\title{
On Stability of a Third Order of Accuracy Difference Scheme for Hyperbolic Nonlocal BVP with Self-Adjoint Operator
}

\author{
Allaberen Ashyralyev ${ }^{1}$ and Ozgur Yildirim ${ }^{2}$ \\ ${ }^{1}$ Department of Mathematics, Fatih University, Buyukcekmece, 34500 Istanbul, Turkey \\ ${ }^{2}$ Department of Mathematics, Yildiz Technical University, Esenler, 34210 Istanbul, Turkey \\ Correspondence should be addressed to Ozgur Yildirim; ozgury@yildiz.edu.tr
}

Received 13 May 2013; Accepted 20 July 2013

Academic Editor: Abdullah Said Erdogan

Copyright (c) $2013 \mathrm{~A}$. Ashyralyev and O. Yildirim. This is an open access article distributed under the Creative Commons Attribution License, which permits unrestricted use, distribution, and reproduction in any medium, provided the original work is properly cited.

\begin{abstract}
A third order of accuracy absolutely stable difference schemes is presented for nonlocal boundary value hyperbolic problem of the differential equations in a Hilbert space $H$ with self-adjoint positive definite operator $A$. Stability estimates for solution of the difference scheme are established. In practice, one-dimensional hyperbolic equation with nonlocal boundary conditions is considered.
\end{abstract}

\section{Introduction}

In modeling several phenomena of physics, biology, and ecology mathematically, there often arise problems with nonlocal boundary conditions (see [1-5] and the references given therein). Nonlocal boundary value problems have been a major research area in the case when it is impossible to determine the boundary conditions of the unknown function. Over the last few decades, the study of nonlocal boundary value problems is of substantial contemporary interest (see, e.g., [6-14] and the references given therein).

We consider the nonlocal boundary value problem

$$
\begin{gathered}
\frac{d^{2} u(t)}{d t^{2}}+A u(t)=f(t), \quad 0<t<1, \\
u(0)=\alpha u(1)+\varphi, \\
u^{\prime}(0)=\beta u^{\prime}(1)+\psi,
\end{gathered}
$$

for hyperbolic equations in a Hilbert space $H$ with selfadjoint positive definite linear operator $A$ with domain $D(A)$.

A function $u(t)$ is called a solution of problem (1) if the following conditions are satisfied.

(i) $u(t)$ is twice continuously differentiable on the segment $[0,1]$. The derivatives at the endpoints of the segment are understood as the appropriate unilateral derivatives.

(ii) The element $u(t)$ belongs to $D(A)$ for all $t \in[0,1]$ and the function $A u(t)$ is continuous on the segment $[0,1]$.

(iii) $u(t)$ satisfies the equations and the nonlocal boundary conditions (1).

Here, $\varphi(x), \psi(x)(x \in[0,1])$ and $f(t, x)(t, x \in[0,1])$ are smooth functions.

In the study of numerical methods for solving PDEs, stability is an important research area (see [6-27]). Many scientists work on difference schemes for hyperbolic partial differential equations, in which stability was established under the assumption that the magnitudes of the grid steps $\tau$ and $h$ with respect to the time and space variables are connected. This particularly means that $\tau\left\|A_{h}\right\| \rightarrow 0$ when $\tau \rightarrow 0$.

We are interested in studying high order of accuracy unconditionally stable difference schemes for hyperbolic PDEs.

In the present paper, third order of accuracy difference scheme generated by integer power of $A$ for approximately solving nonlocal boundary value problem (1) is presented. 
The stability estimates for solution of the difference scheme are established.

In [8], some results of this paper, without proof, were presented.

The well posedness of nonlocal boundary value problems for parabolic equations, elliptic equations, and equations of mixed types have been studied extensively by many scientists (see, e.g., [11-14, 19-32] and the references therein).

\section{Third Order of Accuracy Difference Scheme Subject to Nonlocal Conditions}

In this section, we obtain stability estimates for the solution of third order of accuracy difference scheme

$$
\begin{gathered}
\tau^{-2}\left(u_{k+1}-2 u_{k}+u_{k-1}\right)+\frac{2}{3} A u_{k}+\frac{1}{6} A\left(u_{k+1}+u_{k-1}\right) \\
+\frac{1}{12} \tau^{2} A^{2} u_{k+1}=f_{k}, \\
f_{k}=\frac{2}{3} f\left(t_{k}\right)+\frac{1}{6}\left(f\left(t_{k+1}\right)+f\left(t_{k-1}\right)\right) \\
-\frac{1}{12} \tau^{2}\left(-A f\left(t_{k+1}\right)+f^{\prime \prime}\left(t_{k+1}\right)\right), \\
t_{k}=k \tau, \quad 1 \leq k \leq N-1, N \tau=1, \\
\left.+\frac{\tau^{2}}{12} A+\frac{\tau^{4}}{144} A^{2}\right) \tau^{-1}\left(u_{1}-u_{0}\right)+\frac{\tau}{2} A u_{0}-\tau f_{1,1} \\
=\beta\left(I-\frac{\tau^{2} A}{12}\right) \\
+\left(\frac{\left.7-\frac{\tau^{2} A}{12}\right) \psi}{6 \tau}\right) \\
\quad \times\left(\frac{7 u_{N}-8 u_{N-1}+u_{N-2}}{2}+\frac{\tau}{3}\left(f_{N}-A u_{N}\right)\right)
\end{gathered}
$$

for numerical solution of nonlocal boundary value problem (1). Here,

$$
f_{1,1}=f(0)+\left(-f(0)+\tau f^{\prime}(0)\right) \frac{1}{2}-2 f^{\prime}(0) \frac{\tau}{6} .
$$

We study the stability of solutions of difference scheme (2) under the following assumption:

$$
|\alpha|+2|\beta|+2|\alpha||\beta|<1
$$

We give a lemma that will be needed in the sequel which was presented in [18]. First, let us present the following operators:

$$
\begin{aligned}
R= & \left(I-\frac{1}{3} \tau^{2} A+i \tau A^{1 / 2} \sqrt{I+\frac{1}{72} \tau^{4} A^{2}}\right) \\
& \times\left(I+\frac{1}{6} \tau^{2} A+\frac{1}{12} \tau^{4} A^{2}\right)^{-1},
\end{aligned}
$$

and its conjugate $\widetilde{R}$,

$$
\begin{aligned}
R_{1}=(- & \frac{5 \tau^{4}}{144} A^{2}+\frac{7 \tau^{6}}{216} A^{3}-i \tau A^{1 / 2} \\
& \left.\times\left(I+\frac{\tau^{2}}{12} A+\frac{\tau^{4}}{144} A^{2}\right) \sqrt{I+\frac{1}{72} \tau^{4} A^{2}}\right) \\
& \times\left(-i \tau A^{1 / 2}\left(\sqrt{I+\frac{1}{72} \tau^{4} A^{2}}\right)\right. \\
& \left.\times\left(I+\frac{\tau^{2}}{12} A+\frac{\tau^{4}}{144} A^{2}\right)\right)^{-1}
\end{aligned}
$$

and its conjugate $\widetilde{R}_{1}$,

$$
\begin{aligned}
& R_{2}=\left(I-\frac{\tau^{2}}{12} A\right)\left(I+\frac{\tau^{2}}{6} A+\frac{\tau^{4}}{12} A^{2}\right) \\
& \times\left(-i A^{1 / 2}\left(I+\frac{\tau^{2}}{12} A+\frac{\tau^{4}}{144} A^{2}\right) \sqrt{I+\frac{1}{72} \tau^{4} A^{2}}\right)^{-1}, \\
& R_{3}=\left(I+\frac{\tau^{2}}{6} A+\frac{\tau^{4}}{12} A^{2}\right) \\
& \times\left(\left(I+\frac{\tau^{2}}{12} A+\frac{\tau^{4}}{144} A^{2}\right)\left(-i \tau A^{1 / 2} \sqrt{I+\frac{1}{72} \tau^{4} A^{2}}\right)\right)^{-1}, \\
& R_{4}=\left(I+\frac{\tau^{2}}{3} A+\frac{\tau^{4}}{9} A^{2}+\frac{\tau^{6}}{72} A^{3}\right) \\
& \times\left(-i A^{1 / 2}\left(\sqrt{I+\frac{1}{72} \tau^{4} A^{2}}\right)\left(I+\frac{\tau^{2}}{6} A+\frac{\tau^{4}}{12} A^{2}\right)\right. \\
& \times\left(I+\frac{\tau^{2}}{6} A+\frac{\tau^{4}}{12} A^{2}\right)^{-1}, \\
& R_{5}=\left.-\frac{\tau^{2}}{2} A-\frac{\tau^{4}}{12} A^{2}+i \tau A^{1 / 2} \sqrt{I+\frac{1}{72} \tau^{4} A^{2}}\right) \\
&\left.\times\left(I+\frac{\tau^{2}}{6} A\right)\right)^{-1}, \\
&
\end{aligned}
$$

and its conjugate $\widetilde{R}_{5}$, and

$$
\begin{aligned}
R_{6}= & \left(I-\frac{1}{3} \tau^{2} A+i \tau A^{1 / 2} \sqrt{I+\frac{1}{72} \tau^{4} A^{2}}\right) \\
& \times\left(\frac{\tau^{2}}{2} A+\frac{\tau^{4}}{12} A^{2}-i \tau A^{1 / 2} \sqrt{I+\frac{1}{72} \tau^{4} A^{2}}\right)^{-1},
\end{aligned}
$$

and its conjugate $\widetilde{R}_{6}$. 
We consider the following operators:

$$
\begin{aligned}
R_{7}= & \frac{(7 R-I)}{6 \tau} \\
= & \left(I-\frac{5}{12} \tau^{2} A+\frac{1}{72} \tau^{4} A^{2}+\frac{7}{6} i \tau A^{1 / 2} \sqrt{I+\frac{1}{72} \tau^{4} A^{2}}\right) \\
& \times \tau^{-1}\left(I+\frac{1}{6} \tau^{2} A+\frac{1}{12} \tau^{4} A^{2}\right)^{-1},
\end{aligned}
$$

and its conjugate $\widetilde{R}_{7}$,

$$
\begin{aligned}
\widetilde{R}_{7}= & \frac{(7 \widetilde{R}-I)}{6 \tau} \\
= & \left(I-\frac{5}{12} \tau^{2} A+\frac{1}{72} \tau^{4} A^{2}-\frac{7}{6} i \tau A^{1 / 2} \sqrt{I+\frac{1}{72} \tau^{4} A^{2}}\right) \\
& \times \tau^{-1}\left(I+\frac{1}{6} \tau^{2} A+\frac{1}{12} \tau^{4} A^{2}\right)^{-1}, \\
R_{8}= & \left(\frac{7 I-2 \tau^{2} A}{6 \tau}\right)\left(I+\frac{\tau^{2} A}{3}+\frac{\tau^{4} A^{2}}{9}+\frac{\tau^{6} A^{3}}{72}\right) \\
& \times \tau^{-1}\left(I+\frac{\tau^{2} A}{6}\right)^{-1}\left(I+\frac{\tau^{2}}{6} A+\frac{\tau^{4}}{12} A^{2}\right)^{-2}, \\
R_{9}= & \left(I-\frac{5}{3} \tau^{2} A+\frac{\tau^{4} A^{2}}{9}\right)\left(I+\frac{\tau^{2} A}{3}+\frac{\tau^{4} A^{2}}{9}+\frac{\tau^{6} A^{3}}{72}\right) \\
& \times \tau^{-1}\left(I+\frac{\tau^{2} A}{6}\right)^{-1}\left(I+\frac{1}{6} \tau^{2} A+\frac{1}{12} \tau^{4} A^{2}\right)^{-3}, \\
R_{10}= & I+\left(\frac{5}{144} \tau^{4} A^{2}-\frac{9}{288} \tau^{6} A^{3}+\frac{9}{1728} \tau^{8} A^{4}\right) \\
& \times\left(i \tau A^{1 / 2} \sqrt{I+\frac{1}{72} \tau^{4} A^{2}}\left(I+\frac{\tau^{2}}{12} A+\frac{\tau^{4}}{144} A^{2}\right)\right)^{-1},
\end{aligned}
$$

and its conjugate $\widetilde{R}_{10}$.

Lemma 1. The following estimates hold:

$$
\begin{aligned}
&\|R\|_{H \rightarrow H} \leq 1,\|\widetilde{R}\|_{H \rightarrow H} \leq 1, \\
&\left\|R_{1}\right\|_{H \rightarrow H} \leq 1,\left\|\widetilde{R}_{1}\right\|_{H \rightarrow H} \leq 1, \\
&\left\|A^{1 / 2} R_{2}\right\|_{H \rightarrow H} \leq 1,\left\|\tau A^{1 / 2} R_{3}\right\|_{H \rightarrow H} \leq 1, \\
&\left\|A^{1 / 2} R_{4}\right\|_{H \rightarrow H} \leq 1,\left\|A^{-1 / 2} R_{5}\right\|_{H \rightarrow H} \leq \tau, \\
&\left\|A^{-1 / 2} \widetilde{R}_{5}\right\|_{H \rightarrow H} \leq \tau,\left\|\tau A^{1 / 2} R_{6}\right\|_{H \rightarrow H} \leq 1, \\
&\left\|\tau A^{1 / 2} \widetilde{R}_{6}\right\|_{H \rightarrow H} \leq 1 .
\end{aligned}
$$

Now let us give, without proof, the second lemma.
Lemma 2. The following estimates hold:

$$
\begin{gathered}
\left\|\left(I+i \tau A^{1 / 2}\right) R\right\|_{H \rightarrow H} \leq 2, \\
\left\|\left(I+i \tau A^{1 / 2}\right) \widetilde{R}\right\|_{H \rightarrow H} \leq 2, \\
\left\|\tau R_{7}\right\|_{H \rightarrow H} \leq 1, \quad\left\|\tau \widetilde{R}_{7}\right\|_{H \rightarrow H} \leq 1, \\
\left\|\frac{1}{3} \tau A^{1 / 2} R^{2}\right\|_{H \rightarrow H} \leq 1, \quad\left\|\frac{1}{3} \tau A^{1 / 2} \widetilde{R}^{2}\right\|_{H \rightarrow H} \leq 1, \\
\left\|\tau R_{8}\right\|_{H \rightarrow H} \leq \frac{7}{6}, \quad\left\|\tau R_{9}\right\|_{H \rightarrow H} \leq 1, \\
\left\|R_{10}\left(I+i \tau A^{1 / 2}\right)^{-1}\right\|_{H \rightarrow H} \leq 2, \\
\left\|\widetilde{R}_{10}\left(I+i \tau A^{1 / 2}\right)^{-1}\right\|_{H \rightarrow H} \leq 2 .
\end{gathered}
$$

Throughout the section, for simplicity, we denote

$$
\begin{aligned}
B_{\tau}= & \beta \frac{1}{2} R_{2}\left(\widetilde{R}_{7} \widetilde{R}_{5}-\frac{\tau A}{3} \widetilde{R}^{2}\right) \widetilde{R}^{N-2} \\
& +\beta \frac{1}{2} R_{2}\left(R_{7} R_{5}-\frac{\tau A}{3} R^{2}\right) R^{N-2} \\
& -\alpha \frac{1}{2}\left[\widetilde{R}_{1} R^{N}-R_{1} \widetilde{R}^{N}\right] \\
& +\alpha \beta \frac{1}{4} \widetilde{R}_{1} R_{2}\left(\widetilde{R}_{7} \widetilde{R}_{5}-\frac{\tau A}{3} \widetilde{R}^{2}\right) R^{N} \widetilde{R}^{N-2} \\
& +\alpha \beta \frac{1}{4} R_{1} R_{2}\left(R_{7} R_{5}-\frac{\tau A}{3} R^{2}\right) \widetilde{R}^{N} R^{N-2} \\
& -\alpha \beta \frac{1}{4} \widetilde{R}_{1} R_{2}\left(R_{7} R_{5}-\frac{\tau A}{3} R^{2}\right) \widetilde{R}^{N} R^{N-2} \\
& -\alpha \beta \frac{1}{4} R_{1} R_{2}\left(\widetilde{R}_{7} \widetilde{R}_{5}-\frac{\tau A}{3} \widetilde{R}^{2}\right) R^{N} \widetilde{R}^{N-2} .
\end{aligned}
$$

Lemma 3. Suppose that assumption (4) holds. Then, the operator $I-B_{\tau}$ has an inverse $T_{\tau}=\left(I-B_{\tau}\right)^{-1}$. From symmetry and positivity properties of operator $A$, the following estimate is satisfied:

$$
\left\|T_{\tau}\right\|_{H \mapsto H} \leq \frac{1}{1-|\alpha|-2|\beta|-2|\alpha||\beta|} .
$$

Proof. Using the definitions of $B_{\tau}, R, \widetilde{R}$, estimates (11), and the following simple estimates,

$$
\begin{gathered}
\left\|\tau A^{1 / 2}\left(I-\frac{\tau^{2}}{12} A\right)\left(I+\frac{\tau^{2}}{12} A+\frac{\tau^{4}}{144} A^{2}\right)^{-1}\right\|_{H \rightarrow H} \leq 12, \\
\left\|\tau A^{1 / 2}\left(I+\frac{1}{12} \tau^{2} A+\frac{1}{144} \tau^{4} A^{2}\right)^{-1}\right\|_{H \rightarrow H} \leq \frac{12 \sqrt{11}}{12+\sqrt{11}},
\end{gathered}
$$


and the triangle inequality, we get

$$
\begin{aligned}
& B_{\tau} \leq|\beta| \frac{1}{2}\left\|A^{1 / 2} R_{2}\right\|_{H \rightarrow H}\left\|\widetilde{R}^{N-2}\right\|_{H \rightarrow H} \\
& \times\left(\left\|\tau \widetilde{R}_{7}\right\|_{H \rightarrow H}\left\|\tau^{-1} A^{-1 / 2} \widetilde{R}_{5}\right\|_{H \rightarrow H}+\left\|\frac{1}{3} \tau A^{1 / 2} \widetilde{R}^{2}\right\|_{H \rightarrow H}\right) \\
& +|\beta| \frac{1}{2}\left\|A^{1 / 2} R_{2}\right\|_{H \rightarrow H} \times\left\|R^{N-2}\right\|_{H \rightarrow H} \\
& \times\left(\left\|\tau R_{7}\right\|_{H \rightarrow H}\left\|\tau^{-1} A^{-1 / 2} R_{5}\right\|_{H \rightarrow H}+\left\|\frac{1}{3} \tau A^{1 / 2} R^{2}\right\|_{H \rightarrow H}\right) \\
& +\alpha \frac{1}{2}\left[\left\|\widetilde{R}_{1}\right\|_{H \rightarrow H}\left\|R^{N}\right\|_{H \rightarrow H}+\left\|R_{1}\right\|_{H \rightarrow H}\left\|\widetilde{R}^{N}\right\|_{H \rightarrow H}\right] \\
& +|\alpha||\beta| \frac{1}{4}\left\|\widetilde{R}_{1}\right\|_{H \rightarrow H}\left\|A^{1 / 2} R_{2}\right\|_{H \rightarrow H} \\
& \times\left\|R^{N}\right\|_{H \rightarrow H}\left\|\widetilde{R}^{N-2}\right\|_{H \rightarrow H} \\
& \times\left(\left\|\tau \widetilde{R}_{7}\right\|_{H \rightarrow H}\left\|\tau^{-1} A^{-1 / 2} \widetilde{R}_{5}\right\|_{H \rightarrow H}+\left\|\frac{1}{3} \tau A^{1 / 2} \widetilde{R}^{2}\right\|_{H \rightarrow H}\right) \\
& +|\alpha||\beta| \frac{1}{4}\left\|R_{1}\right\|_{H \rightarrow H}\left\|A^{1 / 2} R_{2}\right\|_{H \rightarrow H} \\
& \times\left\|\widetilde{R}^{N}\right\|_{H \rightarrow H}\left\|R^{N-2}\right\|_{H \rightarrow H} \\
& \times\left(\left\|\tau R_{7}\right\|_{H \rightarrow H}\left\|\tau^{-1} A^{-1 / 2} R_{5}\right\|_{H \rightarrow H}+\left\|\frac{1}{3} \tau A^{1 / 2} R^{2}\right\|_{H \rightarrow H}\right) \\
& +|\alpha||\beta| \frac{1}{4}\left\|\widetilde{R}_{1}\right\|_{H \rightarrow H}\left\|A^{1 / 2} R_{2}\right\|_{H \rightarrow H} \\
& \times\left\|\widetilde{R}^{N}\right\|_{H \rightarrow H}\left\|R^{N-2}\right\|_{H \rightarrow H} \\
& \times\left(\left\|\tau R_{7}\right\|_{H \rightarrow H}\left\|\tau^{-1} A^{-1 / 2} R_{5}\right\|_{H \rightarrow H}+\left\|\frac{1}{3} \tau A^{1 / 2} R^{2}\right\|_{H \rightarrow H}\right) \\
& +|\alpha||\beta| \frac{1}{4}\left\|R_{1}\right\|_{H \rightarrow H}\left\|A^{1 / 2} R_{2}\right\|_{H \rightarrow H} \\
& \times\left\|R^{N}\right\|_{H \rightarrow H}\left\|\widetilde{R}^{N-2}\right\|_{H \rightarrow H} \\
& \times\left(\left\|\tau \widetilde{R}_{7}\right\|_{H \rightarrow H}\left\|\tau^{-1} A^{-1 / 2} \widetilde{R}_{5}\right\|_{H \rightarrow H}+\left\|\frac{1}{3} \tau A^{1 / 2} \widetilde{R}^{2}\right\|_{H \rightarrow H}\right)
\end{aligned}
$$$$
\leq q
$$

where

$$
q=|\alpha|+2|\beta|+2|\alpha||\beta|
$$

Since $q<1$, the operator $I-B_{\tau}$ has a bounded inverse and

$$
\left\|\left(I-B_{\tau}\right)^{-1}\right\|_{H \rightarrow H} \leq \frac{1}{1-q}=\frac{1}{1-|\alpha|-2|\beta|-2|\alpha||\beta|}
$$

Lemma 3 is proved.
Now, let us obtain formula for the solution of problem (2). Using the results of [18], one can obtain the following formula:

$$
\begin{gathered}
u_{0}=\mu, \\
u_{1}=\left(I+\frac{\tau^{2}}{12} A+\frac{\tau^{4}}{144} A^{2}\right)^{-1} \\
\times\left(\left(I-\frac{5}{12} \tau^{2} A+\frac{\tau^{4}}{144} A^{2}\right) \mu\right. \\
+\frac{1}{\left.\left.u_{k}=\frac{\tau^{2}}{2} A\right) \omega+\tau^{2} f_{1,1}\right),} \\
+\frac{1}{2}\left[\widetilde{R}_{10} R^{k}-R_{10} \widetilde{R}^{k}\right] \mu+\frac{1}{2}\left[\widetilde{R}^{k}-R^{k}\right] R_{2} \omega \\
R_{3} \tau^{2} f_{1,1}+\frac{1}{2} R_{4} \sum_{s=1}^{k-1}\left[\widetilde{R}^{k-s}-R^{k-s}\right] f_{s} \tau^{2}
\end{gathered}
$$

for the solution of difference scheme

$$
\begin{gathered}
\tau^{-2}\left(u_{k+1}-2 u_{k}+u_{k-1}\right)+\frac{2}{3} A u_{k}+\frac{1}{6} A\left(u_{k+1}+u_{k-1}\right) \\
+\frac{1}{12} \tau^{2} A^{2} u_{k+1}=f_{k} \\
f_{k}=\frac{2}{3} f\left(t_{k}\right)+\frac{1}{6}\left(f\left(t_{k+1}\right)+f\left(t_{k-1}\right)\right) \\
\quad-\frac{1}{12} \tau^{2}\left(-A f\left(t_{k+1}\right)+f^{\prime \prime}\left(t_{k+1}\right)\right) \\
t_{k}=k \tau, \quad 1 \leq k \leq N-1, N \tau=1 \\
\left(I+\frac{\tau^{2}}{12} A+\frac{\tau^{4}}{144} A^{2}\right) \tau^{-1}\left(u_{1}-u_{0}\right)+\frac{\tau}{2} A u_{0}-\tau f_{1,1} \\
=\left(I-\frac{\tau^{2} A}{12}\right) \omega .
\end{gathered}
$$

Applying formula (19) and nonlocal boundary conditions

$$
u_{0}=\alpha u_{N}+\varphi \text {, }
$$

$$
\omega=\beta\left(\frac{7 u_{N}-8 u_{N-1}+u_{N-2}}{6 \tau}+\frac{\tau}{3}\left(f_{N}-A u_{N}\right)\right)+\psi,
$$


one can write

$$
\begin{aligned}
& \mu=\alpha\left\{\frac{1}{2}\left[\widetilde{R}_{10} R^{N}-R_{10} \widetilde{R}^{N}\right] \mu+\frac{1}{2}\left[\widetilde{R}^{N}-R^{N}\right] R_{2} \omega\right. \\
& +\frac{1}{2}\left[\widetilde{R}^{N}-R^{N}\right] R_{3} \tau^{2} f_{1,1} \\
& \left.+\frac{1}{2} R_{4} \sum_{s=1}^{N-1}\left[\widetilde{R}^{N-s}-R^{N-s}\right] f_{s} \tau^{2}\right\}+\varphi, \\
& \omega=\beta\left\{\frac { 1 } { 2 } \left[\left(R_{7} R_{5}-\frac{\tau A}{3} R^{2}\right) \widetilde{R}_{10} R^{N-2}\right.\right. \\
& \left.-\left(\widetilde{R}_{7} \widetilde{R}_{5}-\frac{\tau A}{3} \widetilde{R}^{2}\right) R_{10} \widetilde{R}^{N-2}\right] \mu \\
& +\frac{1}{2}\left[\left(\widetilde{R}_{7} \widetilde{R}_{5}-\frac{\tau A}{3} \widetilde{R}^{2}\right) \widetilde{R}^{N-2}\right. \\
& \left.-\left(R_{7} R_{5}-\frac{\tau A}{3} R^{2}\right) R^{N-2}\right] R_{2} \omega \\
& +\frac{1}{2}\left[\left(\widetilde{R}_{7} \widetilde{R}_{5}-\frac{\tau A}{3} \widetilde{R}^{2}\right) \widetilde{R}^{N-2}\right. \\
& \left.-\left(R_{7} R_{5}-\frac{\tau A}{3} R^{2}\right) R^{N-2}\right] R_{3} \tau^{2} f_{1,1} \\
& +\frac{\tau}{3} f_{N}+\frac{1}{2} R_{8} f_{N-1} \tau^{2}+\frac{1}{2} R_{9} f_{N-2} \tau^{2} \\
& +\frac{1}{2} R_{4} \tau \sum_{s=1}^{N-3}\left[\left(\widetilde{R}_{7} \widetilde{R}_{5}-\frac{\tau A}{3} \widetilde{R}^{2}\right) \widetilde{R}^{N-2-s}\right. \\
& \left.\left.-\left(R_{7} R_{5}-\frac{\tau A}{3} R^{2}\right) R^{N-2-s}\right] f_{s} \tau\right\}+\psi .
\end{aligned}
$$

Using formulas in (22), we obtain

$$
\begin{aligned}
\mu= & T_{\tau}\left\{\left[\alpha \left(\frac{1}{2}\left(\widetilde{R}^{N}-R^{N}\right) R_{3} \tau^{2} f_{1,1}\right.\right.\right. \\
& \left.\left.+\frac{1}{2} R_{4} \tau \sum_{s=1}^{N-1}\left(\widetilde{R}^{N-s}-R^{N-s}\right) f_{s} \tau\right)+\varphi\right] \\
& \times\left[I-\frac{1}{2}\left(\left(\widetilde{R}_{7} \widetilde{R}_{5}-\frac{\tau A}{3} \widetilde{R}^{2}\right) \widetilde{R}^{N-2}\right.\right. \\
& \left.\left.-\left(R_{7} R_{5}-\frac{\tau A}{3} R^{2}\right) R^{N-2}\right) R_{2}\right]+\left[\alpha \frac{1}{2}\left(\widetilde{R}^{N}-R^{N}\right) R_{2}\right] \\
& \times\left[\beta \frac { 1 } { 2 } \left\{\left(\left(\widetilde{R}_{7} \widetilde{R}_{5}-\frac{\tau A}{3} \widetilde{R}^{2}\right) \widetilde{R}^{N-2}\right.\right.\right. \\
& \left.-\left(R_{7} R_{5}-\frac{\tau A}{3} R^{2}\right) R^{N-2}\right) \times R_{3} \tau^{2} f_{1,1}+\frac{2 \tau}{3} f_{N}+R_{8} f_{N-1} \tau^{2} \\
& +R_{9} f_{N-2} \tau^{2}+R_{4} \tau \sum_{s=1}^{N-3}\left[\left(\widetilde{R}_{7} \widetilde{R}_{5}-\frac{\tau A}{3} \widetilde{R}^{2}\right) \widetilde{R}^{N-2-s}\right. \\
& \left.\left.\left.\left.-\left(R_{7} R_{5}-\frac{\tau A}{3} R^{2}\right) \times R^{N-2-s}\right] f_{s} \tau\right\}+\psi\right]\right\}
\end{aligned}
$$

$$
\begin{aligned}
& \omega=T_{\tau}\left\{\left[I-\alpha \frac{1}{2}\left(\widetilde{R}_{10} R^{N}-R_{10} \widetilde{R}^{N}\right)\right]\right. \\
& \times\left[\beta \frac { 1 } { 2 } \left\{\left(\left(\widetilde{R}_{7} \widetilde{R}_{5}-\frac{\tau A}{3} \widetilde{R}^{2}\right) \widetilde{R}^{N-2}\right.\right.\right. \\
& \left.-\left(R_{7} R_{5}-\frac{\tau A}{3} R^{2}\right) R^{N-2}\right) \times R_{3} \tau^{2} f_{1,1}+\frac{2 \tau}{3} f_{N}+R_{8} f_{N-1} \tau^{2} \\
& +R_{9} f_{N-2} \tau^{2}+R_{4} \tau \times \sum_{s=1}^{N-3}\left(\left(\widetilde{R}_{7} \widetilde{R}_{5}-\frac{\tau A}{3} \widetilde{R}^{2}\right) \widetilde{R}^{N-2-s}\right. \\
& \left.\left.\left.-\left(R_{7} R_{5}-\frac{\tau A}{3} R^{2}\right) \times R^{N-2-s}\right) f_{s} \tau\right\}+\psi\right] \\
& +\frac{1}{2}\left[\left(R_{7} R_{5}-\frac{\tau A}{3} R^{2}\right) \widetilde{R}_{10} R^{N-2}+\left(\widetilde{R}_{7} \widetilde{R}_{5}-\frac{\tau A}{3} \widetilde{R}^{2}\right) R_{10} \widetilde{R}^{N-2}\right] \\
& \times\left[\alpha \left(\frac{1}{2}\left(\widetilde{R}^{N}-R^{N}\right) R_{3} \tau^{2} f_{1,1}\right.\right. \\
& \left.\left.\left.+\frac{1}{2} R_{4} \tau \sum_{s=1}^{N-1}\left(\widetilde{R}^{N-s}-R^{N-s}\right) f_{s} \tau\right)+\varphi\right]\right\} .
\end{aligned}
$$

So, formulas (19) and (23) give a solution of problem (2).

Unfortunately, the estimates for $\max _{1 \leq k \leq N}\left\|u_{k}\right\|_{H}$, $\max _{1 \leq k \leq N}\left\|A^{1 / 2} u_{k}\right\|_{H}$, and $\max _{1 \leq k \leq N}\left\|A u_{k}\right\|_{H}$ cannot be obtained under the conditions

$$
\begin{gathered}
\max _{1 \leq k \leq N}\left\|u_{k}\right\|_{H} \\
\leq M\left\{\sum_{s=1}^{N-1}\left\|A^{-1 / 2} f_{s}\right\|_{H} \tau+\|\varphi\|_{H}+\left\|A^{-1 / 2} \psi\right\|_{H}\right. \\
\left.+\tau\left\|A^{-1 / 2} f_{1,1}\right\|_{H}\right\}, \\
\max _{1 \leq k \leq N}\left\|A^{1 / 2} u_{k}\right\|_{H} \\
\leq M\left\{\sum_{s=1}^{N-1}\left\|f_{s}\right\|_{H} \tau+\left\|A^{1 / 2} \varphi\right\|_{H}+\|\psi\|_{H}+\tau\left\|f_{1,1}\right\|_{H}\right\}, \\
\max _{1 \leq k \leq N}\left\|A u_{k}\right\|_{H} \\
\leq M\left\{\sum_{s=2}^{N-1}\left\|f_{s}-f_{s-1}\right\|_{H}+\left\|f_{1}\right\|_{H}+\|A \varphi\|_{H}\right. \\
\left.+\left\|A^{1 / 2} \psi\right\|_{H}+\tau\left\|A^{1 / 2} f_{1,1}\right\|_{H}\right\} .
\end{gathered}
$$


Theorem 4. Suppose that assumption (4) holds and $\varphi \in$ $D\left(A^{3 / 2}\right), \quad \psi \in D\left(A^{1 / 2}\right)$. Then, for solution of difference scheme (2), the following stability estimates hold:

$\max _{1 \leq k \leq N}\left\|u_{k}\right\|_{H}$

$$
\begin{gathered}
\leq M\left\{\sum_{s=1}^{N-1}\left\|A^{-1 / 2} f_{s}\right\|_{H} \tau+\left\|\left(I+i \tau A^{1 / 2}\right) \varphi\right\|_{H}\right. \\
\left.+\left\|A^{-1 / 2} \psi\right\|_{H}+\tau\left\|A^{-1 / 2} f_{1,1}\right\|_{H}\right\},
\end{gathered}
$$

$$
\begin{gathered}
\max _{1 \leq k \leq N}\left\|A^{1 / 2} u_{k}\right\|_{H} \\
\leq M\left\{\sum_{s=1}^{N-1}\left\|f_{s}\right\|_{H} \tau+\left\|A^{1 / 2}\left(I+i \tau A^{1 / 2}\right) \varphi\right\|_{H}\right. \\
\left.+\|\psi\|_{H}+\tau\left\|f_{1,1}\right\|_{H}\right\},
\end{gathered}
$$

$\max _{1 \leq k \leq N}\left\|A u_{k}\right\|_{H}$

$$
\begin{gathered}
\leq M\left\{\sum_{s=2}^{N-1}\left\|f_{s}-f_{s-1}\right\|_{H}+\left\|f_{1}\right\|_{H}+\left\|A\left(I+i \tau A^{1 / 2}\right) \varphi\right\|_{H}\right. \\
\left.+\left\|A^{1 / 2} \psi\right\|_{H}+\tau\left\|A^{1 / 2} f_{1,1}\right\|_{H}\right\},
\end{gathered}
$$

where $M$ does not depend on $\tau, \varphi, \psi, f_{1,1}(x)$, and $f_{s}(x), 1 \leq$ $s \leq N-1$.

Proof. Using formulas in (23) and estimates (11), (12), and (14), we obtain

$$
\begin{aligned}
& \left\|\left(I+i \tau A^{1 / 2}\right) \mu\right\|_{H} \leq\left\|T_{\tau}\right\|_{H \rightarrow H} \\
& \times\left\{\left[| \alpha | \left(\frac { 1 } { 2 } \left(\left\|\left(I+i \tau A^{1 / 2}\right) \widetilde{R}^{N}\right\|_{H \rightarrow H}\right.\right.\right.\right. \\
& \left.+\left\|\left(I+i \tau A^{1 / 2}\right) R^{N}\right\|_{H \rightarrow H}\right) \\
& \times\left\|\tau A^{1 / 2} R_{3}\right\|_{H \rightarrow H} \tau\left\|A^{-1 / 2} f_{1,1}\right\|_{H} \\
& +\frac{1}{2}\left\|\tau A^{1 / 2} R_{4}\right\|_{H \rightarrow H} \\
& \times \sum_{s=1}^{N-1}\left(\left\|\left(I+i \tau A^{1 / 2}\right) \widetilde{R}^{N-s}\right\|_{H \rightarrow H}\right. \\
& \left.\quad+\left\|\left(I+i \tau A^{1 / 2}\right) R^{N-s}\right\|_{H \rightarrow H}\right) \\
& \left.\left.\times\left\|A^{-1 / 2} f_{s}\right\|_{H \rightarrow H} \tau\right)+\left\|\left(I+i \tau A^{1 / 2}\right) \varphi\right\|_{H}\right]
\end{aligned}
$$

$$
\begin{aligned}
& \times\left[1+\frac{1}{2}\left(\left(\left\|\tau \widetilde{R}_{7}\right\|_{H \rightarrow H}\left\|\tau^{-1} A^{-1 / 2} \widetilde{R}_{5}\right\|_{H \rightarrow H}\right.\right.\right. \\
& \left.+\left\|\frac{1}{3} \tau A^{1 / 2} \widetilde{R}^{2}\right\|_{H \rightarrow H}\right) \\
& \times\left\|\widetilde{R}^{N-2}\right\|_{H \rightarrow H} \\
& +\left(\left\|\tau R_{7}\right\|_{H \rightarrow H}\left\|\tau^{-1} A^{-1 / 2} R_{5}\right\|_{H \rightarrow H}\right. \\
& \left.+\left\|\frac{1}{3} \tau A^{1 / 2} R^{2}\right\|_{H \rightarrow H}\right) \\
& \left.\times\left\|R^{N-2}\right\|_{H \rightarrow H}\right) \\
& \left.\times\left\|A^{1 / 2} R_{2}\right\|_{H \rightarrow H}\right] \\
& +|\alpha| \frac{1}{2}\left(\left\|\left(I+i \tau A^{1 / 2}\right) \widetilde{R}^{N}\right\|_{H \rightarrow H}\right. \\
& \left.+\left\|\left(I+i \tau A^{1 / 2}\right) R^{N}\right\|_{H \rightarrow H}\right) \\
& \times\left\|A^{1 / 2} R_{2}\right\|_{H \rightarrow H} \\
& \times\left[| \beta | \frac { 1 } { 2 } \left\{\left(\left(\left\|\tau \widetilde{R}_{7}\right\|_{H \rightarrow H}\left\|\tau^{-1} A^{-1 / 2} \widetilde{R}_{5}\right\|_{H \rightarrow H}\right.\right.\right.\right. \\
& \left.+\left\|\frac{1}{3} \tau A^{1 / 2} \widetilde{R}^{2}\right\|_{H \rightarrow H}\right) \\
& \times\left\|\widetilde{R}^{N-2}\right\|_{H \rightarrow H} \\
& +\left(\left\|\tau R_{7}\right\|_{H \rightarrow H}\left\|\tau^{-1} A^{-1 / 2} R_{5}\right\|_{H \rightarrow H}\right. \\
& \left.+\left\|\frac{1}{3} \tau A^{1 / 2} R^{2}\right\|_{H \rightarrow H}\right) \\
& \left.\times\left\|R^{N-2}\right\|_{H \rightarrow H}\right) \\
& \times\left\|\tau A^{1 / 2} R_{3}\right\|_{H \rightarrow H} \tau\left\|A^{-1 / 2} f_{1,1}\right\|_{H} \\
& +\frac{2 \tau}{3}\left\|A^{-1 / 2} f_{N}\right\|_{H} \\
& +\left\|\tau R_{8}\right\|_{H \rightarrow H}\left\|A^{-1 / 2} f_{N-1}\right\|_{H} \tau \\
& +\left\|\tau R_{9}\right\|_{H \rightarrow H}\left\|A^{-1 / 2} f_{N-2}\right\|_{H} \tau \\
& +\left\|\tau A^{1 / 2} R_{4}\right\|_{H \rightarrow H} \\
& \times \sum_{s=1}^{N-3}\left(\left(\left\|\tau \widetilde{R}_{7}\right\|_{H \rightarrow H}\right.\right. \\
& \times\left\|\tau^{-1} A^{-1 / 2} \widetilde{R}_{5}\right\|_{H \rightarrow H} \\
& \left.+\left\|\frac{1}{3} \tau A^{1 / 2} \widetilde{R}^{2}\right\|_{H \rightarrow H}\right) \\
& \times\left\|\widetilde{R}^{N-2-s}\right\|_{H \rightarrow H}
\end{aligned}
$$




$$
\begin{aligned}
& +\left(\left\|\tau R_{7}\right\|_{H \rightarrow H}\right.
\end{aligned}
$$

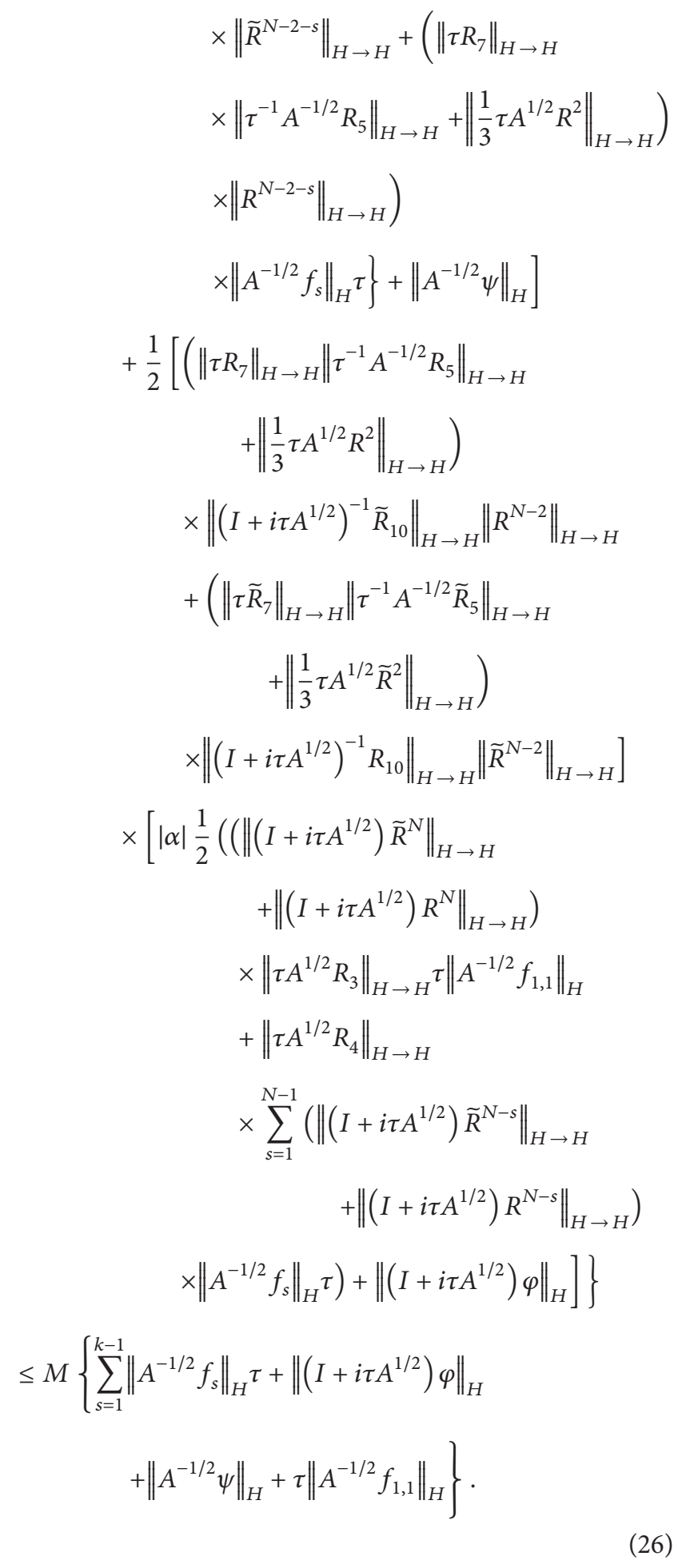

$$
\begin{aligned}
& \left.\times\left\|\tau^{-1} A^{-1 / 2} R_{5}\right\|_{H \rightarrow H}+\left\|\frac{1}{3} \tau A^{1 / 2} R^{2}\right\|_{H \rightarrow H}\right) \\
& \left.\times\left\|R^{N-2-s}\right\|_{H \rightarrow H}\right) \\
& \left.\left.\left.\times\left\|A^{-1 / 2} f_{s}\right\|_{H} \tau\right\}+\left\|A^{-1 / 2} \psi\right\|_{H}\right]\right\} \\
& \leq M\left\{\sum_{s=1}^{k-1}\left\|A^{-1 / 2} f_{s}\right\|_{H} \tau+\left\|\left(I+i \tau A^{1 / 2}\right) \varphi\right\|_{H}\right. \\
& \left.+\left\|A^{-1 / 2} \psi\right\|_{H}+\tau\left\|A^{-1 / 2} f_{1,1}\right\|_{H}\right\}, \\
& \left\|A^{-1 / 2} \omega\right\|_{H} \leq\left\|T_{\tau}\right\|_{H \rightarrow H} \\
& \times\left\{\left[1+|\alpha| \frac{1}{2}\left(\left\|\left(I+i \tau A^{1 / 2}\right)^{-1} \widetilde{R}_{10}\right\|_{H \rightarrow H}\right.\right.\right. \\
& \times\left\|\left(I+i \tau A^{1 / 2}\right) R^{N}\right\|_{H \rightarrow H} \\
& +\left\|\left(I+i \tau A^{1 / 2}\right)^{-1} R_{10}\right\|_{H \rightarrow H} \\
& \left.\left.\times\left\|\left(I+i \tau A^{1 / 2}\right) \widetilde{R}^{N}\right\|_{H \rightarrow H}\right)\right] \\
& \times\left[| \beta | \frac { 1 } { 2 } \left\{\left(\left(\left\|\tau \widetilde{R}_{7}\right\|_{H \rightarrow H}\left\|\tau^{-1} A^{-1 / 2} \widetilde{R}_{5}\right\|_{H \rightarrow H}\right.\right.\right.\right. \\
& \left.+\left\|\frac{1}{3} \tau A^{1 / 2} \widetilde{R}^{2}\right\|_{H \rightarrow H}\right) \\
& \times\left\|\widetilde{R}^{N-2}\right\|_{H \rightarrow H} \\
& +\left(\left\|\tau R_{7}\right\|_{H \rightarrow H}\left\|\tau^{-1} A^{-1 / 2} R_{5}\right\|_{H \rightarrow H}\right. \\
& \left.+\left\|\frac{1}{3} \tau A^{1 / 2} R^{2}\right\|_{H \rightarrow H}\right) \\
& \left.\times\left\|R^{N-2}\right\|_{H \rightarrow H}\right) \\
& \times\left\|\tau A^{1 / 2} R_{3}\right\|_{H \rightarrow H}\left\|A^{-1 / 2} f_{1,1}\right\|_{H} \tau \\
& +\frac{2}{3}\left\|A^{-1 / 2} f_{N}\right\|_{H} \tau \\
& +\left\|\tau R_{8}\right\|_{H \rightarrow H}\left\|A^{-1 / 2} f_{N-1}\right\|_{H} \tau \\
& +\left\|\tau R_{9}\right\|_{H \rightarrow H}\left\|A^{-1 / 2} f_{N-2}\right\|_{H} \tau \\
& +\left\|\tau A^{1 / 2} R_{4}\right\|_{H \rightarrow H} \\
& \times \sum_{s=1}^{N-3}\left(\left(\left\|\tau \widetilde{R}_{7}\right\|_{H \rightarrow H}\right.\right. \\
& \left.\times\left\|\tau^{-1} A^{-1 / 2} \widetilde{R}_{5}\right\|_{H \rightarrow H}+\left\|\frac{1}{3} \tau A^{1 / 2} \widetilde{R}^{2}\right\|_{H \rightarrow H}\right) \\
& \text { Applying } A^{1 / 2} \text { to formulas in (23), we get } \\
& \left\|A^{1 / 2}\left(I+i \tau A^{1 / 2}\right) \mu\right\|_{H} \leq\left\|T_{\tau}\right\|_{H \rightarrow H} \\
& \times\left\{\left[| \alpha | \left(\frac { 1 } { 2 } \left(\left\|\left(I+i \tau A^{1 / 2}\right) \widetilde{R}^{N}\right\|_{H \rightarrow H}\right.\right.\right.\right. \\
& \left.+\left\|\left(I+i \tau A^{1 / 2}\right) R^{N}\right\|_{H \rightarrow H}\right)
\end{aligned}
$$




$$
\begin{aligned}
& \times\left\|\tau A^{1 / 2} R_{3}\right\|_{H \rightarrow H} \tau\left\|f_{1,1}\right\|_{H} \\
& +\frac{1}{2}\left\|\tau A^{1 / 2} R_{4}\right\|_{H \rightarrow H} \\
& \times \sum_{s=1}^{N-1}\left(\left\|\left(I+i \tau A^{1 / 2}\right) \widetilde{R}^{N-s}\right\|_{H \rightarrow H}\right. \\
& \left.+\left\|\left(I+i \tau A^{1 / 2}\right) R^{N-s}\right\|_{H \rightarrow H}\right) \\
& \left.\left.\times\left\|f_{s}\right\|_{H \rightarrow H^{\tau}} \tau\right)+\left\|A^{1 / 2}\left(I+i \tau A^{1 / 2}\right) \varphi\right\|_{H}\right] \\
& \times\left[1+\frac{1}{2}\left(\left(\left\|\tau \widetilde{R}_{7}\right\|_{H \rightarrow H}\left\|\tau^{-1} A^{-1 / 2} \widetilde{R}_{5}\right\|_{H \rightarrow H}\right.\right.\right. \\
& \left.+\left\|\frac{1}{3} \tau A^{1 / 2} \widetilde{R}^{2}\right\|_{H \rightarrow H}\right) \\
& \times\left\|\widetilde{R}^{N-2}\right\|_{H \rightarrow H}+\left(\left\|\tau R_{7}\right\|_{H \rightarrow H}\left\|\tau^{-1} A^{-1 / 2} R_{5}\right\|_{H \rightarrow H}\right. \\
& \left.\left.+\left\|\frac{1}{3} \tau A^{1 / 2} R^{2}\right\|_{H \rightarrow H}\right)\left\|R^{N-2}\right\|_{H \rightarrow H}\right) \\
& \left.\times\left\|A^{1 / 2} R_{2}\right\|_{H \rightarrow H}\right] \\
& +|\alpha| \frac{1}{2}\left(\left\|\left(I+i \tau A^{1 / 2}\right) \widetilde{R}^{N}\right\|_{H \rightarrow H}\right. \\
& \left.+\left\|\left(I+i \tau A^{1 / 2}\right) R^{N}\right\|_{H \rightarrow H}\right) \times\left\|A^{1 / 2} R_{2}\right\|_{H \rightarrow H} \\
& \times\left[| \beta | \frac { 1 } { 2 } \left\{\left(\left(\left\|\tau \widetilde{R}_{7}\right\|_{H \rightarrow H}\left\|\tau^{-1} A^{-1 / 2} \widetilde{R}_{5}\right\|_{H \rightarrow H}\right.\right.\right.\right. \\
& \left.+\left\|\frac{1}{3} \tau A^{1 / 2} \widetilde{R}^{2}\right\|_{H \rightarrow H}\right) \\
& \times\left\|\widetilde{R}^{N-2}\right\|_{H \rightarrow H}+\left(\left\|\tau R_{7}\right\|_{H \rightarrow H}\left\|\tau^{-1} A^{-1 / 2} R_{5}\right\|_{H \rightarrow H}\right. \\
& \left.\left.+\left\|\frac{1}{3} \tau A^{1 / 2} R^{2}\right\|_{H \rightarrow H}\right)\left\|R^{N-2}\right\|_{H \rightarrow H}\right) \\
& \times\left\|\tau A^{1 / 2} R_{3}\right\|_{H \rightarrow H} \tau\left\|f_{1,1}\right\|_{H}+\frac{2 \tau}{3}\left\|f_{N}\right\|_{H} \\
& +\left\|\tau R_{8}\right\|_{H \rightarrow H}\left\|f_{N-1}\right\|_{H} \tau+\left\|\tau R_{9}\right\|_{H \rightarrow H} \\
& \times\left\|f_{N-2}\right\|_{H} \tau+\left\|\tau A^{1 / 2} R_{4}\right\|_{H \rightarrow H} \\
& \times \sum_{s=1}^{N-3}\left(\left(\left\|\tau \widetilde{R}_{7}\right\|_{H \rightarrow H}\left\|\tau^{-1} A^{-1 / 2} \widetilde{R}_{5}\right\|_{H \rightarrow H}\right.\right. \\
& \left.+\left\|\frac{1}{3} \tau A^{1 / 2} \widetilde{R}^{2}\right\|_{H \rightarrow H}\right) \\
& \times\left\|\widetilde{R}^{N-2-s}\right\|_{H \rightarrow H} \\
& +\left(\left\|\tau R_{7}\right\|_{H \rightarrow H}\left\|\tau^{-1} A^{-1 / 2} R_{5}\right\|_{H \rightarrow H}\right.
\end{aligned}
$$$$
\begin{gathered}
\left.+\left\|\frac{1}{3} \tau A^{1 / 2} R^{2}\right\|_{H \rightarrow H}\right) \\
\left.\times\left\|R^{N-2-s}\right\|_{H \rightarrow H}\right) \\
\left.\left.\left.\times\left\|f_{s}\right\|_{H} \tau\right\}+\|\psi\|_{H}\right]\right\} \\
\leq M\left\{\sum_{s=1}^{k-1}\left\|f_{s}\right\|_{H} \tau+\left\|A^{1 / 2}\left(I+i \tau A^{1 / 2}\right) \varphi\right\|_{H}\right. \\
\left.+\|\psi\|_{H}+\tau\left\|f_{1,1}\right\|_{H}\right\}
\end{gathered}
$$$$
\|\omega\|_{H} \leq\left\|T_{\tau}\right\|_{H \rightarrow H}
$$$$
\times\left\{\left[1+|\alpha| \frac{1}{2}\left(\left\|\left(I+i \tau A^{1 / 2}\right)^{-1} \widetilde{R}_{10}\right\|_{H \rightarrow H}\right.\right.\right.
$$$$
\times\left\|\left(I+i \tau A^{1 / 2}\right) R^{N}\right\|_{H \rightarrow H}
$$$$
+\left\|\left(I+i \tau A^{1 / 2}\right)^{-1} R_{10}\right\|_{H \rightarrow H}
$$$$
\left.\left.\times\left\|\left(I+i \tau A^{1 / 2}\right) \widetilde{R}^{N}\right\|_{H \rightarrow H}\right)\right]
$$$$
\times\left[| \beta | \frac { 1 } { 2 } \left\{\left(\left(\left\|\tau \widetilde{R}_{7}\right\|_{H \rightarrow H}\left\|\tau^{-1} A^{-1 / 2} \widetilde{R}_{5}\right\|_{H \rightarrow H}\right.\right.\right.\right.
$$$$
\left.+\left\|\frac{1}{3} \tau A^{1 / 2} \widetilde{R}^{2}\right\|_{H \rightarrow H}\right)
$$$$
\times\left\|\widetilde{R}^{N-2}\right\|_{H \rightarrow H}
$$$$
+\left(\left\|\tau R_{7}\right\|_{H \rightarrow H}\left\|\tau^{-1} A^{-1 / 2} R_{5}\right\|_{H \rightarrow H}\right.
$$$$
\left.+\left\|\frac{1}{3} \tau A^{1 / 2} R^{2}\right\|_{H \rightarrow H}\right)
$$$$
\left.\times\left\|R^{N-2}\right\|_{H \rightarrow H}\right)
$$$$
\times\left\|\tau A^{1 / 2} R_{3}\right\|_{H \rightarrow H}\left\|f_{1,1}\right\|_{H} \tau+\frac{2}{3}\left\|f_{N}\right\|_{H} \tau
$$$$
+\left\|\tau R_{8}\right\|_{H \rightarrow H}\left\|f_{N-1}\right\|_{H} \tau
$$$$
+\left\|\tau R_{9}\right\|_{H \rightarrow H}\left\|f_{N-2}\right\|_{H} \tau+\left\|A^{1 / 2} R_{4}\right\|_{H \rightarrow H}
$$$$
\times \sum_{s=1}^{N-3}\left(\left(\left\|\tau \widetilde{R}_{7}\right\|_{H \rightarrow H}\left\|\tau^{-1} A^{-1 / 2} \widetilde{R}_{5}\right\|_{H \rightarrow H}\right.\right.
$$$$
\left.+\left\|\frac{1}{3} \tau A^{1 / 2} \widetilde{R}^{2}\right\|_{H \rightarrow H}\right)
$$$$
\times\left\|\widetilde{R}^{N-2-s}\right\|_{H \rightarrow H}+\left(\left\|\tau R_{7}\right\|_{H \rightarrow H}\right.
$$$$
\left.\times\left\|\tau^{-1} A^{-1 / 2} R_{5}\right\|_{H \rightarrow H}+\left\|\frac{1}{3} \tau A^{1 / 2} R^{2}\right\|_{H \rightarrow H}\right)
$$ 
Abstract and Applied Analysis

9

$$
\begin{aligned}
& \left.\left.\left.\times\left\|R^{N-2-s}\right\|_{H \rightarrow H}\right)\left\|f_{s}\right\|_{H} \tau\right\}+\|\psi\|_{H}\right] \\
& +\frac{1}{2}\left[\left(\left\|\tau R_{7}\right\|_{H \rightarrow H}\left\|\tau^{-1} A^{-1 / 2} R_{5}\right\|_{H \rightarrow H}\right.\right. \\
& \left.+\left\|\frac{1}{3} \tau A^{1 / 2} R^{2}\right\|_{H \rightarrow H}\right) \\
& \times\left\|\left(I+i \tau A^{1 / 2}\right)^{-1} \widetilde{R}_{10}\right\|_{H \rightarrow H}\left\|R^{N-2}\right\|_{H \rightarrow H} \\
& +\left(\left\|\tau \widetilde{R}_{7}\right\|_{H \rightarrow H}\left\|\tau^{-1} A^{-1 / 2} \widetilde{R}_{5}\right\|_{H \rightarrow H}\right. \\
& \left.+\left\|\frac{1}{3} \tau A^{1 / 2} \widetilde{R}^{2}\right\|_{H \rightarrow H}\right) \\
& \left.\times\left\|\left(I+i \tau A^{1 / 2}\right)^{-1} R_{10}\right\|_{H \rightarrow H}\left\|\widetilde{R}^{N-2}\right\|_{H \rightarrow H}\right] \\
& \times\left[| \alpha | \left(\frac { 1 } { 2 } \left(\left\|\left(I+i \tau A^{1 / 2}\right) \widetilde{R}^{N}\right\|_{H \rightarrow H}\right.\right.\right. \\
& \left.+\left\|\left(I+i \tau A^{1 / 2}\right) R^{N}\right\|_{H \rightarrow H}\right) \\
& \times\left\|\tau A^{1 / 2} R_{3}\right\|_{H \rightarrow H} \tau\left\|f_{1,1}\right\|_{H} \\
& +\frac{1}{2}\left\|\tau A^{1 / 2} R_{4}\right\|_{H \rightarrow H} \\
& \times \sum_{s=1}^{N-1}\left(\left\|\left(I+i \tau A^{1 / 2}\right) \widetilde{R}^{N-s}\right\|_{H \rightarrow H}\right. \\
& \left.+\left\|\left(I+i \tau A^{1 / 2}\right) R^{N-s}\right\|_{H \rightarrow H}\right) \\
& \left.\left.\left.\times\left\|f_{s}\right\|_{H} \tau\right)+\left\|A^{1 / 2}\left(I+i \tau A^{1 / 2}\right) \varphi\right\|_{H}\right]\right\} \\
& \leq M\left\{\sum_{s=1}^{k-1}\left\|f_{s}\right\|_{H} \tau+\left\|A^{1 / 2}\left(I+i \tau A^{1 / 2}\right) \varphi\right\|_{H}\right. \\
& \left.+\|\psi\|_{H}+\tau\left\|f_{1,1}\right\|_{H}\right\} .
\end{aligned}
$$

Now, applying Abel's formula to (23), we obtain the following formulas:

$$
\begin{aligned}
\mu=T_{\tau}\left\{\left[\alpha \left(\frac{1}{2}\left(\widetilde{R}^{N}-R^{N}\right) R_{3} \tau^{2} f_{1,1}\right.\right.\right. \\
+\frac{1}{2} R_{4} \tau^{2}\left(\sum_{s=2}^{N-1}\left(R_{6} R^{N-s}-\widetilde{R}_{6} \widetilde{R}^{N-s}\right)\right. \\
\quad \times\left(f_{s}-f_{s-1}\right)+\left(\widetilde{R}_{6}-R_{6}\right) f_{N-1} \\
\left.\left.\left.\quad-\left(\widetilde{R}_{6} \widetilde{R}^{N-1}-R_{6} R^{N-1}\right) f_{1}\right)\right)+\varphi\right]
\end{aligned}
$$

$$
\begin{aligned}
& \times\left[I-\frac{1}{2}\left(\left(\widetilde{R}_{7} \widetilde{R}_{5}-\frac{\tau A}{3} \widetilde{R}^{2}\right) \widetilde{R}^{N-2}\right.\right. \\
& \left.\left.-\left(R_{7} R_{5}-\frac{\tau A}{3} R^{2}\right) R^{N-2}\right) R_{2}\right] \\
& +\alpha \frac{1}{2}\left(\widetilde{R}^{N}-R^{N}\right) R_{2} \\
& \times\left[\beta \left\{\frac { 1 } { 2 } \left(\left(\widetilde{R}_{7} \widetilde{R}_{5}-\frac{\tau A}{3} \widetilde{R}^{2}\right) \widetilde{R}^{N-2}\right.\right.\right. \\
& \left.-\left(R_{7} R_{5}-\frac{\tau A}{3} R^{2}\right) R^{N-2}\right) \\
& \times R_{3} \tau^{2} f_{1,1}+\frac{\tau}{3} f_{N}+\frac{1}{2} R_{8} f_{N-1} \tau^{2}+\frac{1}{2} R_{9} f_{N-2} \tau^{2} \\
& +R_{4} \frac{1}{2} \tau^{2}\left(\sum _ { s = 2 } ^ { N - 3 } \left(R_{6}\left(R_{7} R_{5}-\frac{\tau A}{3} R^{2}\right) R^{N-2-s}\right.\right. \\
& \left.-\widetilde{R}_{6}\left(\widetilde{R}_{7} \widetilde{R}_{5}-\frac{\tau A}{3} \widetilde{R}^{2}\right) \widetilde{R}^{N-2-s}\right) \\
& \times\left(f_{s}-f_{s-1}\right) \\
& +\left(\widetilde{R}_{6}\left(\widetilde{R}_{7} \widetilde{R}_{5}-\frac{\tau A}{3} \widetilde{R}^{2}\right)\right. \\
& \left.-R_{6}\left(R_{7} R_{5}-\frac{\tau A}{3} R^{2}\right)\right) f_{N-3} \\
& -\left(\widetilde{R}_{6}\left(\widetilde{R}_{7} \widetilde{R}_{5}-\frac{\tau A}{3} \widetilde{R}^{2}\right) \widetilde{R}^{N-3}\right. \\
& \left.-R_{6}\left(R_{7} R_{5}-\frac{\tau A}{3} R^{2}\right) R^{N-3}\right) \\
& \left.\left.\left.\left.\times f_{1}\right)\right\}+\psi\right]\right\}
\end{aligned}
$$




$$
\begin{aligned}
& \times\left(f_{s}-f_{s-1}\right) \\
& +\left(\widetilde{R}_{6}\left(\widetilde{R}_{7} \widetilde{R}_{5}-\frac{\tau A}{3} \widetilde{R}^{2}\right)\right. \\
& \left.-R_{6}\left(R_{7} R_{5}-\frac{\tau A}{3} R^{2}\right)\right) f_{N-3} \\
& -\left(\widetilde{R}_{6}\left(\widetilde{R}_{7} \widetilde{R}_{5}-\frac{\tau A}{3} \widetilde{R}^{2}\right) \widetilde{R}^{N-3}\right. \\
& -R_{6}\left(R_{7} R_{5}-\frac{\tau A}{3} R^{2}\right) \\
& \left.\left.\left.\left.\times R^{N-3}\right) f_{1}\right)\right\}+\psi\right] \\
& +\frac{1}{2}\left[\left(R_{7} R_{5}-\frac{\tau A}{3} R^{2}\right) \widetilde{R}_{1} R^{N-2}\right. \\
& \left.-\left(\widetilde{R}_{7} \widetilde{R}_{5}-\frac{\tau A}{3} \widetilde{R}^{2}\right) R_{1} \widetilde{R}^{N-2}\right] \\
& \times\left[\alpha \left(\frac{1}{2}\left(\widetilde{R}^{N}-R^{N}\right) R_{3} \tau^{2} f_{1,1}\right.\right. \\
& +\frac{1}{2} R_{4} \tau^{2}\left(\sum_{s=2}^{N-1}\left(R_{6} R^{N-s}-\widetilde{R}_{6} \widetilde{R}^{N-s}\right)\right. \\
& \times\left(f_{s}-f_{s-1}\right) \\
& +\left(\widetilde{R}_{6}-R_{6}\right) f_{N-1} \\
& -\left(\widetilde{R}_{6} \widetilde{R}^{N-1}-R_{6} R^{N-1}\right) \\
& \left.\left.\left.\left.\times f_{1}\right)\right)+\varphi\right]\right\} \text {. }
\end{aligned}
$$

Next, let us obtain the estimates for $\left\|A\left(I+i \tau A^{1 / 2}\right) \mu\right\|_{H}$ and $\left\|A^{1 / 2} \omega\right\|_{H}$. First, applying $A$ to formula (28) and using estimates (11), (12), and (14) and the triangle inequality, one can obtain

$$
\begin{aligned}
\left\|A\left(I+i \tau A^{1 / 2}\right) \mu\right\|_{H} \leq\left\|T_{\tau}\right\|_{H \rightarrow H} \\
\times\left\{\left[| \alpha | \left(\frac { 1 } { 2 } \left(\left\|\left(I+i \tau A^{1 / 2}\right) \widetilde{R}^{N}\right\|_{H \rightarrow H}\right.\right.\right.\right. \\
\left.+\left\|\left(I+i \tau A^{1 / 2}\right) R^{N}\right\|_{H \rightarrow H}\right) \\
\times\left\|\tau A^{1 / 2} R_{3}\right\|_{H \rightarrow H}\left\|A^{1 / 2} f_{1,1}\right\|_{H} \tau \\
+\frac{1}{2}\left\|\tau A^{1 / 2} R_{4}\right\|_{H \rightarrow H} \\
\times\left(\sum _ { s = 2 } ^ { N - 1 } \left(\left\|\tau A^{1 / 2} R_{6}\right\|_{H \rightarrow H}\right.\right. \\
\times\left\|\left(I+i \tau A^{1 / 2}\right) R^{N-s}\right\|_{H \rightarrow H}
\end{aligned}
$$

$$
\begin{aligned}
& +\left\|\tau A^{1 / 2} \widetilde{R}_{6}\right\|_{H \rightarrow H} \\
& \left.\times\left\|\left(I+i \tau A^{1 / 2}\right) \widetilde{R}^{N-s}\right\|_{H \rightarrow H}\right) \\
& \times\left\|f_{s}-f_{s-1}\right\|_{H} \\
& +\left(\left\|\tau A^{1 / 2}\left(I+i \tau A^{1 / 2}\right) \widetilde{R}_{6}\right\|_{H \rightarrow H}\right. \\
& \left.+\left\|\tau A^{1 / 2}\left(I+i \tau A^{1 / 2}\right) R_{6}\right\|_{H \rightarrow H}\right) \times\left\|f_{N-1}\right\|_{H} \\
& +\left(\left\|\tau A^{1 / 2} \widetilde{R}_{6}\right\|_{H \rightarrow H}\right. \\
& \times\left\|\left(I+i \tau A^{1 / 2}\right) \widetilde{R}^{N-1}\right\|_{H \rightarrow H} \\
& +\left\|\tau A^{1 / 2} R_{6}\right\|_{H \rightarrow H} \\
& \left.\times\left\|\left(I+i \tau A^{1 / 2}\right) R^{N-1}\right\|_{H \rightarrow H}\right) \\
& \left.\left.\left.\times\left\|f_{1}\right\|_{H}\right)\right)+\left\|A\left(I+i \tau A^{1 / 2}\right) \varphi\right\|_{H}\right] \\
& \times\left[1+\frac{1}{2}\left(\left(\left\|\tau \widetilde{R}_{7}\right\|_{H \rightarrow H}\left\|\tau^{-1} A^{-1 / 2} \widetilde{R}_{5}\right\|_{H \rightarrow H}\right.\right.\right. \\
& \left.+\left\|\frac{1}{3} \tau A^{1 / 2} \widetilde{R}^{2}\right\|_{H \rightarrow H}\right)\left\|\widetilde{R}^{N-2}\right\|_{H \rightarrow H} \\
& +\left(\left\|\tau R_{7}\right\|_{H \rightarrow H}\left\|\tau^{-1} A^{-1 / 2} R_{5}\right\|_{H \rightarrow H}\right. \\
& \left.+\left\|\frac{1}{3} \tau A^{1 / 2} R^{2}\right\|_{H \rightarrow H}\right) \\
& \left.\left.\times\left\|R^{N-2}\right\|_{H \rightarrow H}\right)\left\|A^{1 / 2} R_{2}\right\|_{H \rightarrow H}\right] \\
& +|\alpha| \frac{1}{2}\left(\left\|\left(I+i \tau A^{1 / 2}\right) \widetilde{R}^{N}\right\|_{H \rightarrow H}\right. \\
& \left.+\left\|\left(I+i \tau A^{1 / 2}\right) R^{N}\right\|_{H \rightarrow H}\right)\left\|A^{1 / 2} R_{2}\right\|_{H \rightarrow H} \\
& \times\left[| \beta | \frac { 1 } { 2 } \left\{\left(\left(\left\|\tau \widetilde{R}_{7}\right\|_{H \rightarrow H}\left\|\tau^{-1} A^{-1 / 2} \widetilde{R}_{5}\right\|_{H \rightarrow H}\right.\right.\right.\right. \\
& \left.+\left\|\frac{1}{3} \tau A^{1 / 2} \widetilde{R}^{2}\right\|_{H \rightarrow H}\right)\left\|\widetilde{R}^{N-2}\right\|_{H \rightarrow H} \\
& +\left(\left\|\tau R_{7}\right\|_{H \rightarrow H}\left\|\tau^{-1} A^{-1 / 2} R_{5}\right\|_{H \rightarrow H}\right. \\
& \left.+\left\|\frac{1}{3} \tau A^{1 / 2} R^{2}\right\|_{H \rightarrow H}\right) \\
& \left.\times\left\|R^{N-2}\right\|_{H \rightarrow H}\right) \times\left\|\tau A^{1 / 2} R_{3}\right\|_{H \rightarrow H} \\
& \times \tau\left\|A^{1 / 2} f_{1,1}\right\|_{H}+\frac{2 \tau}{3}\left\|A^{1 / 2} f_{N}\right\|_{H} \\
& +\left\|\tau R_{8}\right\|_{H \rightarrow H}\left\|A^{1 / 2} f_{N-1}\right\|_{H} \tau \\
& +\left\|\tau R_{9}\right\|_{H \rightarrow H}\left\|A^{1 / 2} f_{N-2}\right\|_{H} \tau
\end{aligned}
$$




$$
\begin{aligned}
& +\left\|\tau A^{1 / 2} R_{4}\right\|_{H \rightarrow H} \\
& \left.+\left\|\frac{1}{3} \tau A^{1 / 2} R^{2}\right\|_{H \rightarrow H}\right) \\
& \times\left(\sum _ { s = 2 } ^ { N - 3 } \left(\left\|\tau A^{1 / 2} R_{6}\right\|_{H \rightarrow H}\right.\right. \\
& \times\left(\left\|\tau R_{7}\right\|_{H \rightarrow H}\right. \\
& \times\left\|\tau^{-1} A^{-1 / 2} R_{5}\right\|_{H \rightarrow H} \\
& \left.+\left\|\frac{1}{3} \tau A^{1 / 2} R^{2}\right\|_{H \rightarrow H}\right) \\
& \times\left\|R^{N-2-s}\right\|_{H \rightarrow H} \\
& +\left\|\tau A^{1 / 2} \widetilde{R}_{6}\right\|_{H \rightarrow H} \\
& \times\left(\left\|\tau \widetilde{R}_{7}\right\|_{H \rightarrow H}\right. \\
& \times\left\|\tau^{-1} A^{-1 / 2} \widetilde{R}_{5}\right\|_{H \rightarrow H} \\
& \left.+\left\|\frac{1}{3} \tau A^{1 / 2} \widetilde{R}^{2}\right\|_{H \rightarrow H}\right) \quad\left\|A^{1 / 2} \omega\right\|_{H} \leq\left\|T_{\tau}\right\|_{H \rightarrow H} \\
& \left.\times\left\|\widetilde{R}^{N-2-s}\right\|_{H \rightarrow H}\right)\left\|f_{s}-f_{s-1}\right\|_{H} \\
& +\left(\left\|\tau A^{1 / 2} \widetilde{R}_{6}\right\|_{H \rightarrow H}\right. \\
& \times\left(\left\|\tau \widetilde{R}_{7}\right\|_{H \rightarrow H}\right. \\
& \times\left\|\tau^{-1} A^{-1 / 2} \widetilde{R}_{5}\right\|_{H \rightarrow H} \\
& \left.+\left\|\frac{1}{3} \tau A^{1 / 2} \widetilde{R}^{2}\right\|_{H \rightarrow H}\right) \\
& +\left\|\tau A^{1 / 2} R_{6}\right\|_{H \rightarrow H} \\
& \times\left(\left\|\tau R_{7}\right\|_{H \rightarrow H}\right. \\
& \times\left\|\tau^{-1} A^{-1 / 2} R_{5}\right\|_{H \rightarrow H} \\
& \left.\left.+\left\|\frac{1}{3} \tau A^{1 / 2} R^{2}\right\|_{H \rightarrow H}\right)\right) \\
& \times\left\{\left[1+|\alpha| \frac{1}{2}\left(\left\|\left(I+i \tau A^{1 / 2}\right)^{-1} \widetilde{R}_{10}\right\|_{H \rightarrow H}\right.\right.\right. \\
& \times\left\|\left(I+i \tau A^{1 / 2}\right) R^{N}\right\|_{H \rightarrow H} \\
& +\left\|\left(I+i \tau A^{1 / 2}\right)^{-1} R_{10}\right\|_{H \rightarrow H} \\
& \left.\left.\times\left\|\left(I+i \tau A^{1 / 2}\right) \widetilde{R}^{N}\right\|_{H \rightarrow H}\right)\right] \\
& \times\left[| \beta | \frac { 1 } { 2 } \left\{\left(\left(\left\|\tau \widetilde{R}_{7}\right\|_{H \rightarrow H}\left\|\tau^{-1} A^{-1 / 2} \widetilde{R}_{5}\right\|_{H \rightarrow H}\right.\right.\right.\right. \\
& \left.+\left\|\frac{1}{3} \tau A^{1 / 2} \widetilde{R}^{2}\right\|_{H \rightarrow H}\right)\left\|\widetilde{R}^{N-2}\right\|_{H \rightarrow H} \\
& +\left(\left\|\tau R_{7}\right\|_{H \rightarrow H}\left\|\tau^{-1} A^{-1 / 2} R_{5}\right\|_{H \rightarrow H}\right. \\
& \left.+\left\|\frac{1}{3} \tau A^{1 / 2} R^{2}\right\|_{H \rightarrow H}\right) \\
& \left.\times\left\|R^{N-2}\right\|_{H \rightarrow H}\right) \\
& \times\left\|f_{N-3}\right\|_{H} \\
& +\left(\left\|\tau A^{1 / 2} \widetilde{R}_{6}\right\|_{H \rightarrow H}\right. \\
& \times\left(\left\|\tau \widetilde{R}_{7}\right\|_{H \rightarrow H}\left\|\tau^{-1} A^{-1 / 2} \widetilde{R}_{5}\right\|_{H \rightarrow H}\right. \\
& \left.+\left\|\frac{1}{3} \tau A^{1 / 2} \widetilde{R}^{2}\right\|_{H \rightarrow H}\right) \\
& \times\left\|\widetilde{R}^{N-3}\right\|_{H \rightarrow H}+\left\|\tau A^{1 / 2} R_{6}\right\|_{H \rightarrow H} \\
& \times\left(\left\|\tau R_{7}\right\|_{H \rightarrow H}\left\|\tau^{-1} A^{-1 / 2} R_{5}\right\|_{H \rightarrow H}\right.
\end{aligned}
$$


12

Abstract and Applied Analysis

$$
\begin{aligned}
& \times\left(\left\|\tau R_{7}\right\|_{H \rightarrow H}\left\|\tau^{-1} A^{-1 / 2} R_{5}\right\|_{H \rightarrow H}\right. \\
& \left.+\left\|\frac{1}{3} \tau A^{1 / 2} R^{2}\right\|_{H \rightarrow H}\right) \\
& \times\left\|R^{N-2-s}\right\|_{H \rightarrow H}+\left\|\tau A^{1 / 2} \widetilde{R}_{6}\right\|_{H \rightarrow H} \\
& \times\left(\left\|\tau \widetilde{R}_{7}\right\|_{H \rightarrow H}\left\|\tau^{-1} A^{-1 / 2} \widetilde{R}_{5}\right\|_{H \rightarrow H}\right. \\
& \left.+\left\|\frac{1}{3} \tau A^{1 / 2} \widetilde{R}^{2}\right\|_{H \rightarrow H}\right) \\
& \left.\times\left\|\widetilde{R}^{N-2-s}\right\|_{H \rightarrow H}\right)\left\|f_{s}-f_{s-1}\right\|_{H} \\
& +\left(\left\|\tau A^{1 / 2} \widetilde{R}_{6}\right\|_{H \rightarrow H}\right. \\
& \times\left(\left\|\tau \widetilde{R}_{7}\right\|_{H \rightarrow H}\right. \\
& \times\left\|\tau^{-1} A^{-1 / 2} \widetilde{R}_{5}\right\|_{H \rightarrow H} \\
& \left.+\left\|\frac{1}{3} \tau A^{1 / 2} \widetilde{R}^{2}\right\|_{H \rightarrow H}\right) \\
& +\left\|\tau A^{1 / 2} R_{6}\right\|_{H \rightarrow H} \\
& \times\left(\left\|\tau R_{7}\right\|_{H \rightarrow H}\left\|\tau^{-1} A^{-1 / 2} R_{5}\right\|_{H \rightarrow H}\right. \\
& \left.\left.+\left\|\frac{1}{3} \tau A^{1 / 2} R^{2}\right\|_{H \rightarrow H}\right)\right) \\
& \times\left\|f_{N-3}\right\|_{H} \\
& +\left(\left\|\tau A^{1 / 2} \widetilde{R}_{6}\right\|_{H \rightarrow H}\right. \\
& \times\left(\left\|\tau \widetilde{R}_{7}\right\|_{H \rightarrow H}\left\|\tau^{-1} A^{-1 / 2} \widetilde{R}_{5}\right\|_{H \rightarrow H}\right. \\
& \left.+\left\|\frac{1}{3} \tau A^{1 / 2} \widetilde{R}^{2}\right\|_{H \rightarrow H}\right) \\
& \times\left\|\widetilde{R}^{N-3}\right\|_{H \rightarrow H} \\
& +\left\|\tau A^{1 / 2} R_{6}\right\|_{H \rightarrow H} \\
& \times\left(\left\|\tau R_{7}\right\|_{H \rightarrow H}\left\|\tau^{-1} A^{-1 / 2} R_{5}\right\|_{H \rightarrow H}\right. \\
& \left.+\left\|\frac{1}{3} \tau A^{1 / 2} R^{2}\right\|_{H \rightarrow H}\right) \\
& \left.\left.\left.\times\left\|R^{N-3}\right\|_{H \rightarrow H}\right)\left\|f_{1}\right\|_{H}\right)\right\} \\
& \left.+\left\|A^{1 / 2} \psi\right\|_{H}\right] \\
& +\frac{1}{2}\left[\left(\left\|\tau R_{7}\right\|_{H \rightarrow H}\left\|\tau^{-1} A^{-1 / 2} R_{5}\right\|_{H \rightarrow H}\right.\right.
\end{aligned}
$$

$$
\begin{aligned}
& \left.+\left\|\frac{1}{3} \tau A^{1 / 2} R^{2}\right\|_{H \rightarrow H}\right) \\
& \times\left\|\widetilde{R}_{10}\left(I+i \tau A^{1 / 2}\right)^{-1}\right\|_{H \rightarrow H} \\
& \times\left\|\left(I+i \tau A^{1 / 2}\right) R^{N-2}\right\|_{H \rightarrow H} \\
& +\left(\left\|\tau \widetilde{R}_{7}\right\|_{H \rightarrow H}\left\|\tau^{-1} A^{-1 / 2} \widetilde{R}_{5}\right\|_{H \rightarrow H}\right. \\
& \left.+\left\|\frac{1}{3} \tau A^{1 / 2} \widetilde{R}^{2}\right\|_{H \rightarrow H}\right) \\
& \times\left\|R_{10}\left(I+i \tau A^{1 / 2}\right)^{-1}\right\|_{H \rightarrow H} \\
& \left.\times\left\|\left(I+i \tau A^{1 / 2}\right) \widetilde{R}^{N-2}\right\|_{H \rightarrow H}\right] \\
& \times\left[|\alpha| \frac{1}{2}\left(\left\|\widetilde{R}^{N}\right\|_{H \rightarrow H}+\left\|R^{N}\right\|_{H \rightarrow H}\right)\right. \\
& \times\left\|\tau A^{1 / 2} R_{3}\right\|_{H \rightarrow H} \tau\left\|A^{1 / 2} f_{1,1}\right\|_{H} \\
& +\left\|\tau A^{1 / 2} R_{4}\right\|_{H \rightarrow H} \\
& \times\left(\sum _ { s = 2 } ^ { N - 1 } \left(\left\|\tau A^{1 / 2} R_{6}\right\|_{H \rightarrow H}\left\|R^{N-s}\right\|_{H \rightarrow H}\right.\right. \\
& \left.+\left\|\tau A^{1 / 2} \widetilde{R}_{6}\right\|_{H \rightarrow H}\left\|\widetilde{R}^{N-s}\right\|_{H \rightarrow H}\right) \\
& \times\left\|f_{s}-f_{s-1}\right\|_{H} \\
& +\left(\left\|\tau A^{1 / 2} \widetilde{R}_{6}\right\|_{H \rightarrow H}+\left\|\tau A^{1 / 2} R_{6}\right\|_{H \rightarrow H}\right) \\
& \times\left\|f_{N-1}\right\|_{H \rightarrow H} \\
& +\left(\left\|\tau A^{1 / 2} \widetilde{R}_{6}\right\|_{H \rightarrow H}\left\|\widetilde{R}^{N-1}\right\|_{H \rightarrow H}\right. \\
& \left.+\left\|\tau A^{1 / 2} R_{6}\right\|_{H \rightarrow H}\left\|R^{N-1}\right\|_{H \rightarrow H}\right) \\
& \left.\times\left\|f_{1}\right\|_{H}\right) \\
& \left.\left.+\left\|\left(I+i \tau A^{1 / 2}\right)^{-1}\right\|_{H \rightarrow H}\left\|A\left(I+i \tau A^{1 / 2}\right) \varphi\right\|_{H}\right]\right\} \\
& \leq M\left\{\sum_{s=2}^{N-1}\left\|f_{s}-f_{s-1}\right\|_{H}+\left\|f_{1}\right\|_{H}+\left\|A\left(I+i \tau A^{1 / 2}\right) \varphi\right\|_{H}\right. \\
& \left.+\left\|A^{1 / 2} \psi\right\|_{H}+\tau\left\|A^{1 / 2} f_{1,1}\right\|_{H}\right\} .
\end{aligned}
$$

Now, we will prove estimates (25). Using formula (19), estimates (11), (12), (26), and (27), and the triangle inequality, we obtain

$$
\begin{aligned}
\left\|u_{k}\right\|_{H} \leq & \frac{1}{2}\left(\left\|\widetilde{R}_{10}\left(I+i \tau A^{1 / 2}\right)^{-1}\right\|_{H \rightarrow H}\left\|R^{k}\right\|_{H \rightarrow H}\right. \\
& \left.+\left\|R_{10}\left(I+i \tau A^{1 / 2}\right)^{-1}\right\|_{H \rightarrow H}\left\|\widetilde{R}^{k}\right\|_{H \rightarrow H}\right) \\
\times & \left\|\left(I+i \tau A^{1 / 2}\right) \mu\right\|_{H}
\end{aligned}
$$


Abstract and Applied Analysis

13

$$
\begin{aligned}
& +\frac{1}{2}\left(\left\|A^{1 / 2} R_{2}\right\|_{H \rightarrow H}\left\|\widetilde{R}^{k}\right\|_{H \rightarrow H}\right. \\
& \left.+\left\|A^{1 / 2} R_{2}\right\|_{H \rightarrow H}\left\|R^{k}\right\|_{H \rightarrow H}\right) \\
& \times\left\|A^{-1 / 2} \omega\right\|_{H} \\
& +\frac{1}{2}\left(\left\|\tau A^{1 / 2} R_{3}\right\|_{H \rightarrow H}\left\|\widetilde{R}^{k}\right\|_{H \rightarrow H}\right. \\
& +\frac{1}{2}\left\|\tau A^{1 / 2} R_{4}\right\|_{H \rightarrow H} \sum_{s=1}^{k-1}\left[\left\|\widetilde{R}^{k-s}\right\|_{H \rightarrow H}+\left\|R^{k-s}\right\|_{H \rightarrow H}\right] \\
& \times\left\|A^{-1 / 2} f_{s}\right\|_{H} \tau \\
& \leq\left\{\begin{array}{l}
N-1 \\
\sum_{s=1}^{k}\left\|A^{-1 / 2} f_{s}\right\|_{H} \tau+\left\|\left(I+i \tau A^{1 / 2}\right) \varphi\right\|_{H}
\end{array}\right) \tau A^{-1 / 2} f_{1,1} \|_{H} \\
& \left.+\left\|A^{-1 / 2} \psi\right\|_{H}+\tau\left\|A^{-1 / 2} f_{1,1}\right\|_{H}\right\}
\end{aligned}
$$

for any $k \geq 2$. Applying $A^{1 / 2}$ to (19), we get

$$
\begin{aligned}
& \left\|A^{1 / 2} u_{k}\right\|_{H} \\
& \leq \frac{1}{2}\left(\left\|\widetilde{R}_{10}\left(I+i \tau A^{1 / 2}\right)^{-1}\right\|_{H \rightarrow H}\left\|R^{k}\right\|_{H \rightarrow H}\right. \\
& \left.+\left\|R_{10}\left(I+i \tau A^{1 / 2}\right)^{-1}\right\|_{H \rightarrow H}\left\|\widetilde{R}^{k}\right\|_{H \rightarrow H}\right) \\
& \times\left\|A^{1 / 2}\left(I+i \tau A^{1 / 2}\right) \mu\right\|_{H} \\
& +\frac{1}{2}\left(\left\|A^{1 / 2} R_{2}\right\|_{H \rightarrow H}\left\|\widetilde{R}^{k}\right\|_{H \rightarrow H}\right. \\
& +\frac{1}{2}\left(\left\|\tau A^{1 / 2} R_{3}\right\|_{H \rightarrow H}\left\|\widetilde{R}^{k}\right\|_{H \rightarrow H}\left\|_{H \rightarrow H}\right\| R^{k} \|_{H \rightarrow H}\right) \times\|\omega\|_{H} \\
& \left.+\left\|\tau A^{1 / 2} R_{3}\right\|_{H \rightarrow H}\left\|R^{k}\right\|_{H \rightarrow H}\right) \times \tau\left\|f_{1,1}\right\|_{H} \\
& +\frac{1}{2}\left\|\tau A^{1 / 2} R_{4}\right\|_{H \rightarrow H} \sum_{s=1}^{k-1}\left(\left\|\widetilde{R}^{k-s}\right\|_{H \rightarrow H}+\left\|R^{k-s}\right\|_{H \rightarrow H}\right) \\
& \times\left\|f_{s}\right\|_{H} \tau \leq M\left\{\sum_{s=1}^{N-1}\left\|f_{s}\right\|_{H} \tau+\left\|A^{1 / 2}\left(I+i \tau A^{1 / 2}\right) \varphi\right\|_{H}+\tau\left\|f_{1,1}\right\|_{H}\right\}
\end{aligned}
$$

for $k \geq 2$. Now, applying Abel's formula to (19), we have

$$
\begin{aligned}
u_{k}= & \frac{1}{2}\left[\widetilde{R}_{1} R^{k}-R_{1} \widetilde{R}^{k}\right] \mu+\frac{1}{2}\left[\widetilde{R}^{k}-R^{k}\right] R_{2} \omega \\
& +\frac{1}{2}\left[\widetilde{R}^{k}-R^{k}\right] R_{3} \tau^{2} f_{1,1} \\
& +\tau^{2} R_{4} \frac{1}{2}\left(\sum_{s=2}^{k-1}\left[R_{6} R^{k-s}-\widetilde{R}_{6} \widetilde{R}^{k-s}\right]\left(f_{s}-f_{s-1}\right)\right. \\
& +\left(\widetilde{R}_{6}-R_{6}\right) f_{k-1} \\
& \left.-\left[\widetilde{R}_{6} \widetilde{R}^{k-1}-R_{6} R^{k-1}\right] f_{1}\right), \quad 2 \leq k \leq N .
\end{aligned}
$$

Applying $A$ to formula (34) and using estimates (11) and (12) and the triangle inequality, we obtain

$$
\begin{aligned}
& \left\|A u_{k}\right\|_{H} \leq \frac{1}{2}\left(\left\|\widetilde{R}_{10}\left(I+i \tau A^{1 / 2}\right)^{-1}\right\|_{H \rightarrow H}\left\|R^{k}\right\|_{H \rightarrow H}\right. \\
& \left.+\left\|R_{10}\left(I+i \tau A^{1 / 2}\right)^{-1}\right\|_{H \rightarrow H}\left\|\widetilde{R}^{k}\right\|_{H \rightarrow H}\right) \\
& \times\left\|A\left(I+i \tau A^{1 / 2}\right) \mu\right\|_{H} \\
& +\frac{1}{2}\left(\left\|A^{1 / 2} R_{2}\right\|_{H \rightarrow H}\left\|\widetilde{R}^{k}\right\|_{H \rightarrow H}\right. \\
& \left.+\left\|A^{1 / 2} R_{2}\right\|_{H \rightarrow H}\left\|R^{k}\right\|_{H \rightarrow H}\right) \\
& \times\left\|A^{1 / 2} \omega\right\|_{H}+\frac{1}{2}\left(\left\|\tau A^{-1 / 2} R_{3}\right\|_{H \rightarrow H}\left\|\widetilde{R}^{k}\right\|_{H \rightarrow H}\right. \\
& \left.+\left\|\tau A^{-1 / 2} R_{3}\right\|_{H \rightarrow H}\left\|R^{k}\right\|_{H \rightarrow H}\right) \\
& \times \tau\left\|A^{1 / 2} f_{1,1}\right\|_{H}+\frac{1}{2}\left\|\tau A^{1 / 2} R_{4}\right\|_{H \rightarrow H} \\
& \times\left(\sum _ { s = 2 } ^ { k - 1 } \left[\left\|\tau A^{1 / 2} R_{6}\right\|_{H \rightarrow H}\left\|R^{k-s}\right\|_{H \rightarrow H}\right.\right. \\
& \left.+\left\|\tau A^{1 / 2} \widetilde{R}_{6}\right\|_{H \rightarrow H}\left\|\widetilde{R}^{k-s}\right\|_{H \rightarrow H}\right] \\
& \times\left\|f_{s}-f_{s-1}\right\|_{H} \\
& +\left(\left\|\tau A^{1 / 2} \widetilde{R}_{6}\right\|_{H \rightarrow H}+\left\|\tau A^{1 / 2} R_{6}\right\|_{H \rightarrow H}\right)\left\|f_{k-1}\right\|_{H} \\
& +\left[\left\|\tau A^{1 / 2} \widetilde{R}_{6}\right\|_{H \rightarrow H}\left\|\widetilde{R}^{k-1}\right\|_{H \rightarrow H}\right. \\
& \left.\left.+\left\|\tau A^{1 / 2} R_{6}\right\|_{H \rightarrow H}\left\|R^{k-1}\right\|_{H \rightarrow H}\right]\left\|f_{1}\right\|_{H}\right)
\end{aligned}
$$




$$
\begin{gathered}
\leq M\left\{\sum_{s=2}^{N-1}\left\|f_{s}-f_{s-1}\right\|_{H}+\left\|f_{1}\right\|_{H}+\left\|A\left(I+i \tau A^{1 / 2}\right) \varphi\right\|_{H}\right. \\
\left.+\left\|A^{1 / 2} \psi\right\|_{H}+\tau\left\|A^{1 / 2} f_{1,1}\right\|_{H}\right\}
\end{gathered}
$$

for $k \geq 2$. Theorem 4 is proved.

Note that the stability estimates obtained previously permit us to get the convergence estimate of difference scheme (2) under the smoothness property of solution (1). Actually, under the condition $u(t) \in C([0,1], H)$, we can obtain the third order of accuracy for the error of difference scheme (2). Since $u^{(6)}(t)=-A^{3} u(t)+A^{2} f(t)-A f^{\prime \prime}(t)+f^{(4)}(t)$, this condition is satisfied under the given data $\varphi \in D\left(A^{3}\right)$, $\psi \in D\left(A^{5 / 2}\right), f^{\prime}(t) \in D\left(A^{2}\right)$, and $f(0) \in D\left(A^{3}\right)$.

Now, let us give application of this abstract result for nonlocal boundary value problem

$$
\begin{gathered}
u_{t t}-\left(a(x) u_{x}\right)_{x}+\delta u=f(t, x), \quad 0<t<1, \quad 0<x<1, \\
u(0, x)=\alpha u(1, x)+\varphi(x), \quad 0 \leq x \leq 1, \\
u_{t}(0, x)=\beta u_{t}(1, x)+\psi(x), \quad 0 \leq x \leq 1, \\
u(t, 0)=u(t, 1), \quad u_{x}(t, 0)=u_{x}(t, 1), \quad 0 \leq t \leq 1
\end{gathered}
$$

for hyperbolic equation. Problem (36) has a unique smooth solution $u(t, x), \delta>0$ and the smooth functions $a(x) \geq$ $a>0(a(0)=a(1), x \in(0,1)), \varphi(x), \psi(x)(x \in[0,1])$, and $f(t, x)(t, x \in[0,1])$. This allows us to reduce mixed problem (36) to nonlocal boundary value problem (1) in a Hilbert space $H=L_{2}[0,1]$ with a self-adjoint positive definite operator $A^{x}$ defined by (36).

The discretization of problem (36) is carried out in two steps. In the first step, let us define the grid space

$$
[0,1]_{h}=\left\{x: x_{r}=r h, 0 \leq r \leq K, K h=1\right\} .
$$

We introduce Hilbert space $L_{2 h}=L_{2}\left([0,1]_{h}\right), W_{2 h}^{1}=$ $W_{2 h}^{1}\left([0,1]_{h}\right)$, and $W_{2 h}^{2}=W_{2 h}^{2}\left([0,1]_{h}\right)$ of the grid functions $\varphi^{h}(x)=\left\{\varphi_{r}\right\}_{1}^{K-1}$ defined on $[0,1]_{h}$, and we assign the difference operator $A_{h}^{x}$ by the formula

$$
A_{h}^{x} \varphi^{h}(x)=\left\{-\left(a(x) \varphi_{\bar{x}}\right)_{x, r}+\delta \varphi_{r}\right\}_{1}^{K-1},
$$

acting in the space of grid functions $\varphi^{h}(x)=\left\{\varphi_{r}\right\}_{0}^{K}$ satisfying the conditions $\varphi_{0}=\varphi_{K}, \varphi_{1}-\varphi_{0}=\varphi_{K}-\varphi_{K-1}$.

With the help of $A_{h}^{x}$, we arrive at the nonlocal boundary value problem

$$
\begin{gathered}
\frac{d^{2} v^{h}(t, x)}{d t^{2}}+A_{h}^{x} v^{h}(t, x)=f^{h}(t, x), \\
0<t<1, \quad x \in[0,1]_{h}, \\
v^{h}(0, x)=\alpha v^{h}(1, x)+\varphi^{h}(x), \quad x \in[0,1]_{h}, \\
v_{t}^{h}(0, x)=\beta v_{t}^{h}(1, x)+\psi^{h}(x), \quad x \in[0,1]_{h}
\end{gathered}
$$

for a system of ordinary differential equations.
In the second step, we replace problem (2) with difference scheme (40)

$$
\begin{aligned}
& \tau^{-2}\left(u_{k+1}^{h}(x)-2 u_{k}^{h}(x)+u_{k-1}^{h}(x)\right)+\frac{2}{3} A_{h}^{x} u_{k}^{h}(x) \\
& +\frac{1}{6} A_{h}^{x}\left(u_{k+1}^{h}(x)+u_{k-1}^{h}(x)\right) \\
& +\frac{1}{12} \tau^{2}\left(A_{h}^{x}\right)^{2} u_{k+1}^{h}(x)=f_{k}^{h}(x), \\
& f_{k}^{h}(x)=\frac{2}{3} f^{h}\left(t_{k}, x\right) \\
& +\frac{1}{6}\left(f^{h}\left(t_{k+1}, x\right)+f^{h}\left(t_{k-1}, x\right)\right) \\
& -\frac{1}{12} \tau^{2}\left(-A f^{h}\left(t_{k+1}, x\right)+f_{t t}^{h}\left(t_{k+1}, x\right)\right), \quad x \in[0,1]_{h}, \\
& t_{k}=k \tau, \quad N \tau=1, \quad 1 \leq k \leq N-1, \\
& u_{0}^{h}(x)=\alpha u_{N}^{h}(x)+\varphi^{h}(x), \quad x \in[0,1]_{h}, \\
& \left(I+\frac{\tau^{2}}{12}\left(A_{h}^{x}\right)+\frac{\tau^{4}}{144}\left(A_{h}^{x}\right)^{2}\right) \tau^{-1}\left(u_{1}^{h}(x)-u_{0}^{h}(x)\right) \\
& +\frac{\tau}{2}\left(A_{h}^{x}\right) \varphi^{h}(x)-\tau f_{1,1}^{h}(x) \\
& =\beta\left(I-\frac{\tau^{2}}{12}\left(A_{h}^{x}\right)\right) \\
& \times\left(\frac{1}{6 \tau}\left(7 u_{N}^{h}(x)-8 u_{N-1}^{h}(x)+u_{N-2}^{h}(x)\right)\right. \\
& \left.+\frac{\tau}{3}\left(f_{N}^{h}(x)-A u_{N}^{h}(x)\right)\right) \\
& +\left(I-\frac{\tau^{2}}{12}\left(A_{h}^{x}\right)\right) \psi^{h}(x), \quad x \in[0,1]_{h}, \\
& f_{1,1}^{h}(x)=\frac{1}{2} f^{h}(0, x)+\frac{\tau}{6} f_{t}^{h}(0, x) \text {. }
\end{aligned}
$$

Theorem 5. Let $\tau$ and $h$ be sufficiently small numbers. Then, the solution of difference scheme (40) satisfies the following stability estimates:

$$
\begin{gathered}
\max _{0 \leq k \leq N}\left\|u_{k}^{h}\right\|_{L_{2 h}}+\max _{0 \leq k \leq N}\left\|u_{k}^{h}\right\|_{W_{2 h}^{1}} \\
\leq M_{1}\left[\max _{1 \leq k \leq N-1}\left\|f_{k}^{h}\right\|_{L_{2 h}}+\left\|\psi^{h}\right\|_{L_{2 h}}\right. \\
\left.+\left\|\varphi^{h}\right\|_{W_{2 h}^{1}}+\tau\left\|\varphi^{h}\right\|_{W_{2 h}^{2}}+\tau\left\|f_{1,1}^{h}\right\|_{L_{2 h}}\right], \\
\max _{1 \leq k \leq N-1}\left\|\tau^{-2}\left(u_{k+1}^{h}-2 u_{k}^{h}+u_{k-1}^{h}\right)\right\|_{L_{2 h}}+\max _{0 \leq k \leq N}\left\|u_{k}^{h}\right\|_{W_{2 h}^{2}} \\
\leq M_{1}\left[\left\|f_{1}^{h}\right\|_{L_{2 h}}+\max _{2 \leq k \leq N-1}\left\|\tau^{-1}\left(f_{k}^{h}-f_{k-1}^{h}\right)\right\|_{L_{2 h}}\right. \\
+\left\|\psi^{h}\right\|_{W_{2 h}^{1}}+\left\|\varphi^{h}\right\|_{W_{2 h}^{2}} \\
\left.+\tau\left\|\varphi^{h}\right\|_{W_{2 h}^{3}}+\tau\left\|f_{1,1}^{h}\right\|_{W_{2 h}^{1}}\right] .
\end{gathered}
$$


Here, $M_{1}$ does not depend on $\tau, h, \varphi^{h}(x), \psi^{h}(x), f_{1,1}^{h}(x)$, and $f_{k}^{h}(x), 1 \leq k<N$.

The proof of Theorem 5 is based on the proof of abstract Theorem 4 and the symmetry property of operator $A_{h}^{x}$ defined by (38).

\section{Acknowledgments}

The authors would like to thank Professor Pavel E. Sobolevskii and reviewers for their helpful comments.

\section{References}

[1] H. Lamb, Hydrodynamics, Cambridge Mathematical Library, Cambridge University Press, Cambridge, UK, 6th edition, 1993.

[2] J. Lighthill, Waves in Fluids, Cambridge University Press, Cambridge, UK, 1978.

[3] J. A. Hudson, The Excitation and Propagation of Elastic Waves, Cambridge Monographs on Mechanics and Applied Mathematics, Cambridge University Press, Cambridge, UK, 1980.

[4] D. S. Jones, Acoustic and Electromagnetic Waves, Oxford Science Publications, The Clarendon Press/Oxford University Press, New York, NY, USA, 1986.

[5] A. Taflove, Computational Electrodynamics: The FiniteDifference Time-Domain Method, Artech House, Boston, Mass, USA, 1995.

[6] A. Ashyralyev and N. Aggez, "Finite difference method for hyperbolic equations with the nonlocal integral condition," Discrete Dynamics in Nature and Society, vol. 2011, Article ID 562385, 15 pages, 2011.

[7] A. Ashyralyev and O. Yildirim, "A note on the second order of accuracy stable difference schemes for the nonlocal boundary value hyperbolic problem," Abstract and Applied Analysis, vol. 2012, Article ID 846582, 29 pages, 2012.

[8] A. Ashyralyev and O. Yildirim, "High order of accuracy stable difference schemes for numerical solutions of NBVP for hyperbolic equations," in Proceedings of the 1st International Conference on Analysis and Applied Mathematics (ICAAM '12), vol. 1470 of AIP Conference Proceedings, pp. 84-87, 2012.

[9] A. Ashyralyev and O. Yildirim, "On multipoint nonlocal boundary value problems for hyperbolic differential and difference equations," Taiwanese Journal of Mathematics, vol. 14, no. 1, pp. 165-194, 2010.

[10] M. E. Koksal, "Recent developments on operator-difference schemes for solving nonlocal BVPs for the wave equation," Discrete Dynamics in Nature and Society, vol. 2011, Article ID 210261, 14 pages, 2011.

[11] Y. Ozdemir and M. Kucukunal, "A note on nonlocal boundary value problems for hyperbolic Schrödinger equations," Abstract and Applied Analysis, vol. 2012, Article ID 687321, 12 pages, 2012.

[12] N. Gordeziani, P. Natalini, and P. E. Ricci, "Finite-difference methods for solution of nonlocal boundary value problems," Computers \& Mathematics with Applications, vol. 50, no. 8-9, pp. 1333-1344, 2005.

[13] J. Martín-Vaquero, A. Queiruga-Dios, and A. H. Encinas, "Numerical algorithms for diffusion-reaction problems with non-classical conditions," Applied Mathematics and Computation, vol. 218, no. 9, pp. 5487-5495, 2012.

[14] A. Ashyralyev and O. Gercek, "On second order of accuracy difference scheme of the approximate solution of nonlocal elliptic-parabolic problems," Abstract and Applied Analysis, vol. 2010, Article ID 705172, 17 pages, 2010.

[15] A. Ashyralyev and P. E. Sobolevskii, "Two new approaches for construction of the high order of accuracy difference schemes for hyperbolic differential equations," Discrete Dynamics in Nature and Society, vol. 2005, no. 2, pp. 183-213, 2005.

[16] P. E. Sobolevskii and L. M. Chebotaryeva, "Approximate solution by method of lines of the Cauchy problem for an abstract hyperbolic equations," Izvestiya Vysshikh Uchebnykh Zavedenij Matematika, vol. 5, no. 1, pp. 103-116, 1977.

[17] A. Ashyralyev, M. E. Koksal, and R. P. Agarwal, "A difference scheme for Cauchy problem for the hyperbolic equation with self-adjoint operator," Mathematical and Computer Modelling, vol. 52, no. 1-2, pp. 409-424, 2010.

[18] A. Ashyralyev and O. Yildirim, "On the numerical solution of hyperbolic IBVP with high order stable difference schemes," Boundary Value Problems, vol. 2013, no. 29, 36 pages, 2013.

[19] A. Ashyralyev and P. E. Sobolevskii, New Difference Schemes for Partial Differential Equations, vol. 148 of Operator Theory: Advances and Applications, Birkhäuser, Basel, Switzerland, 2004.

[20] A. Ashyralyev, G. Judakova, and P. E. Sobolevskii, "A note on the difference schemes for hyperbolic-elliptic equations," Abstract and Applied Analysis, vol. 2006, 13 pages, 2006.

[21] D. Guidetti, B. Karasözen, and S. Piskarev, "Approximation of abstract differential equations," Journal of Mathematical Sciences, vol. 122, no. 2, pp. 3013-3054, 2004.

[22] A. A. Samarskii, V. I. Korzyuk, S. V. Lemeshevsky, and P. P. Matus, "Finite-difference methods for problem of conjugation of hyperbolic and parabolic equations," Mathematical Models \& Methods in Applied Sciences, vol. 10, no. 3, pp. 361-377, 2000.

[23] P. D. Lax and B. Wendroff, "Difference schemes for hyperbolic equations with high order of accuracy," Communications on Pure and Applied Mathematics, vol. 17, pp. 381-398, 1964.

[24] H. O. Fattorini, Second Order Linear Differential Equations in Banach Spaces, vol. 108 of Notas de Matemática, North-Holland, 1985.

[25] S. G. Krein, Linear Differential Equations in a Banach Space, Nauka, Moscow, Russia, 1966.

[26] S. Piskarev and S.-Y. Shaw, "On certain operator families related to cosine operator functions," Taiwanese Journal of Mathematics, vol. 1, no. 4, pp. 3585-3592, 1997.

[27] A. Ashyralyev, "Nonlocal boundary-value problems for abstract parabolic equations: well-posedness in Bochner spaces," Journal of Evolution Equations, vol. 6, no. 1, pp. 1-28, 2006.

[28] R. P. Agarwal, M. Bohner, and V. B. Shakhmurov, "Maximal regular boundary value problems in Banach-valued weighted space," Boundary Value Problems, vol. 2005, no. 1, pp. 9-42, 2005.

[29] T. D. Dzuraev, Boundary Value Problems for Equations of Mixed and Mixed-Composite Types, FAN, Tashkent, Uzbekistan, 1979.

[30] A. V. Gulin, N. I. Ionkin, and V. A. Morozova, "On the stability of a nonlocal two-dimensional difference problem," Differential Equations, vol. 7, no. 7, pp. 970-978, 2001.

[31] D. Gordeziani, H. Meladze, and G. Avalishvili, "On one class of nonlocal in time problems for first-order evolution equations," Zhurnal Obchyslyuval'noï ta Prykladnoï Matematyky, vol. 88, no. 1, pp. 66-78, 2003.

[32] M. S. Salahatdinov, Equations of Mixed-Composite Type, FAN, Tashkent, Uzbekistan, 1974. 


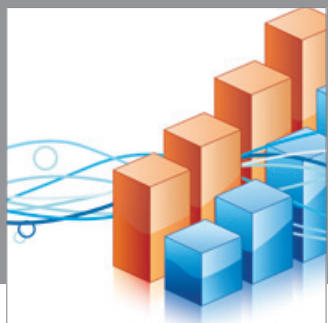

Advances in

Operations Research

mansans

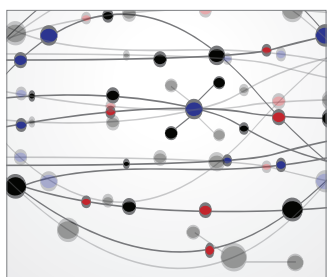

The Scientific World Journal
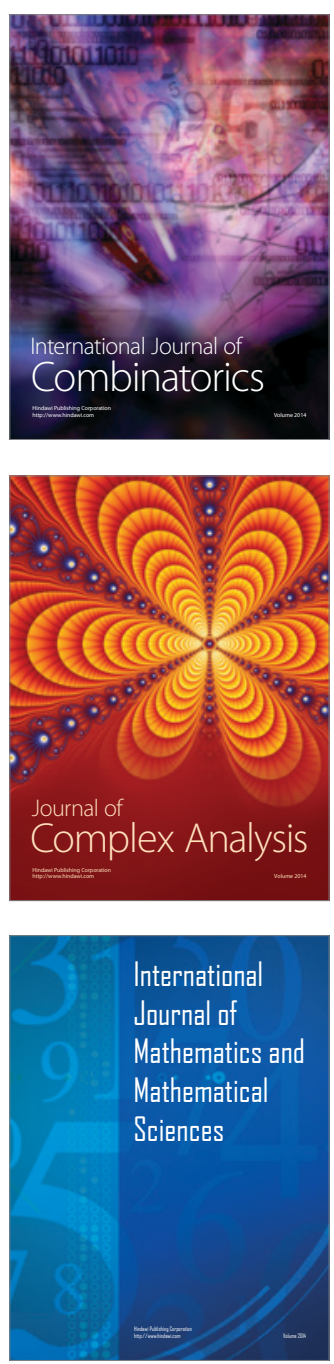
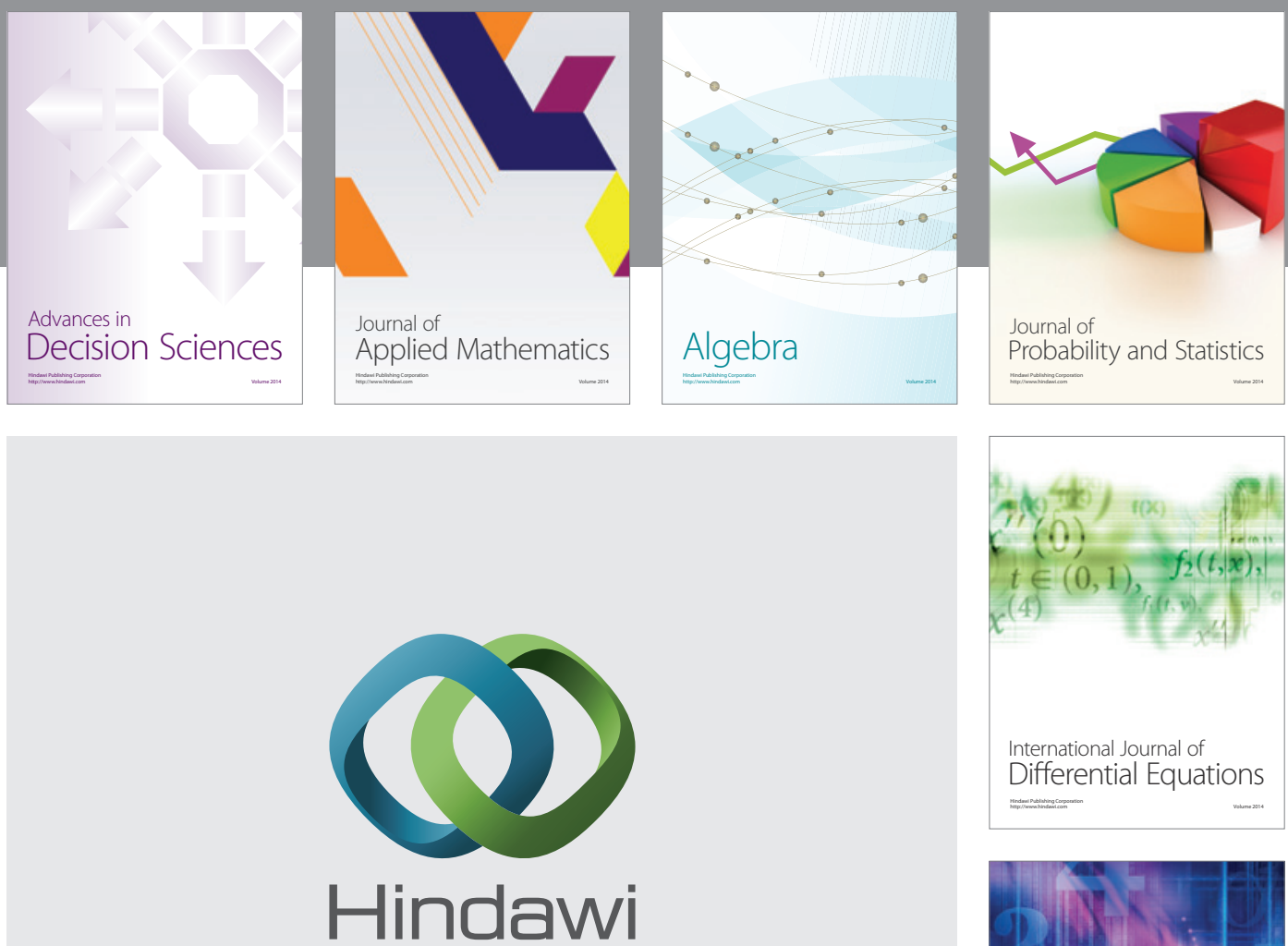

Submit your manuscripts at http://www.hindawi.com
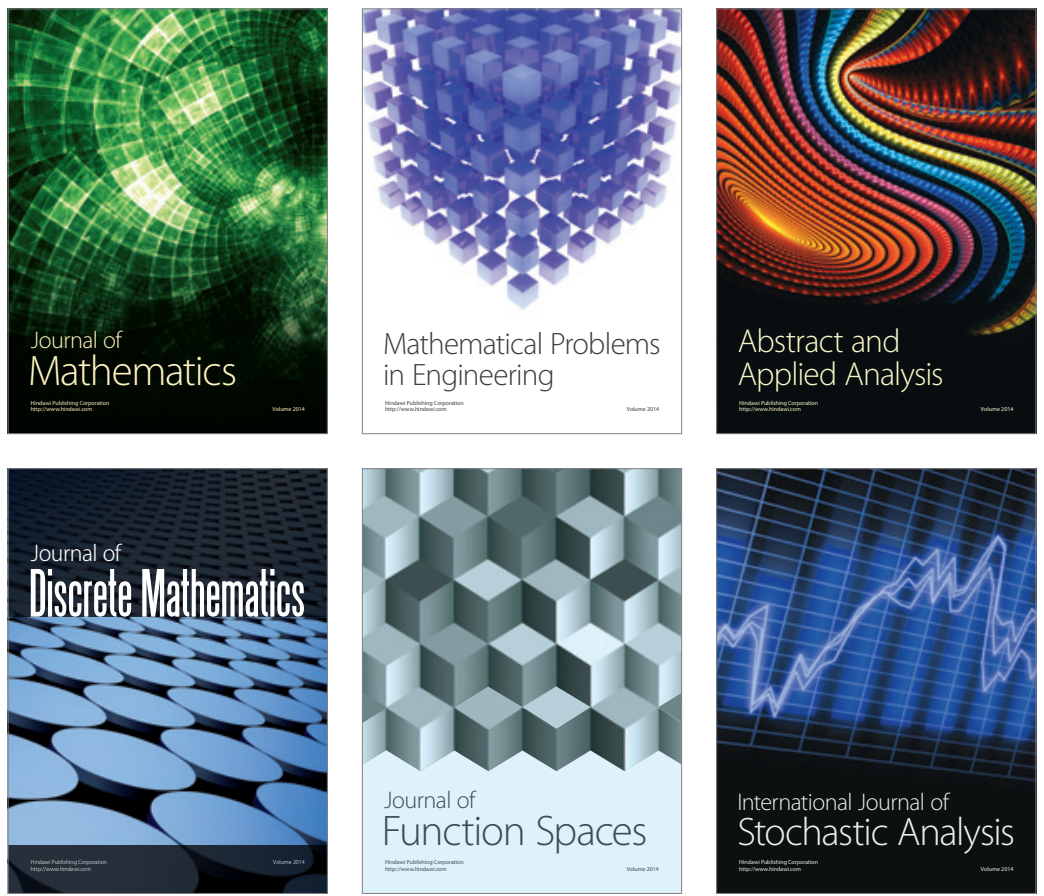

Journal of

Function Spaces

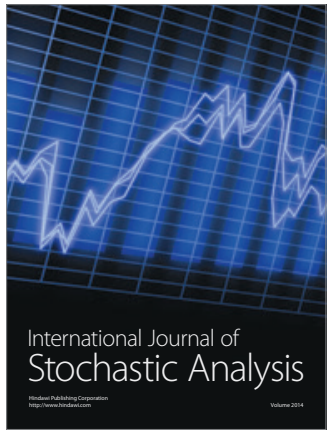

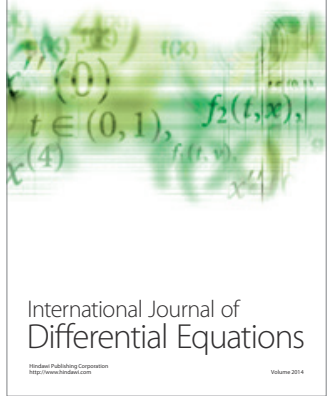
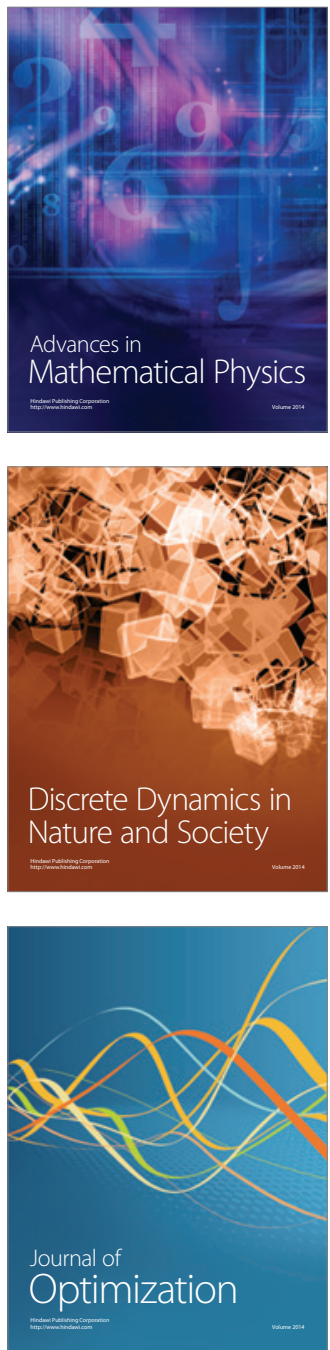